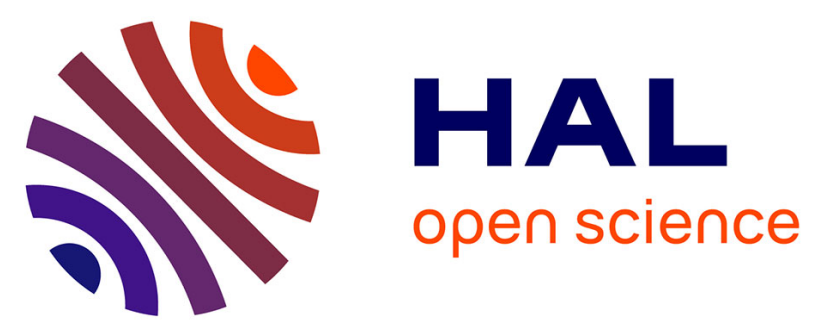

\title{
Throughput-Efficient Super-TDMA MAC Transmission Schedules in Ad Hoc Linear Underwater Acoustic Networks
}

\author{
Said Lmai, Mandar Chitre, Christophe Laot, Sébastien Houcke
}

\section{- To cite this version:}

Said Lmai, Mandar Chitre, Christophe Laot, Sébastien Houcke. Throughput-Efficient Super-TDMA MAC Transmission Schedules in Ad Hoc Linear Underwater Acoustic Networks. IEEE Journal of Oceanic Engineering, 2017, 42 (1), pp.156 - 174. 10.1109/JOE.2016.2537659 . hal-01487046

\author{
HAL Id: hal-01487046 \\ https://hal.science/hal-01487046
}

Submitted on 7 Jun 2021

HAL is a multi-disciplinary open access archive for the deposit and dissemination of scientific research documents, whether they are published or not. The documents may come from teaching and research institutions in France or abroad, or from public or private research centers.
L'archive ouverte pluridisciplinaire HAL, est destinée au dépôt et à la diffusion de documents scientifiques de niveau recherche, publiés ou non, émanant des établissements d'enseignement et de recherche français ou étrangers, des laboratoires publics ou privés. 


\title{
Throughput-efficient super-TDMA MAC transmission schedules in ad hoc linear
}

\section{underwater acoustic networks}

\author{
Said Lmai, Member, IEEE, Mandar Chitre, Senior Member, IEEE, \\ Christophe Laot, Senior Member, IEEE, and Sebastien Houcke, Member, IEEE,
}

\begin{abstract}
Underwater acoustic (UWA) sensor network deployments may be used in many applications for environmental, scientific, military and commercial purposes. Several topologies are in use, but the most widely used topology is linear. Indeed, placing nodes on a single line offers more opportunities in terms of large coverage and high-rate services. The UWA channel is a shared medium. Thus, a medium access control (MAC) protocol is necessary, primarily to regulate and coordinate nodes' access. MAC protocol design should take into consideration large propagation delays to favor better network throughput. Performance of most developed protocols in linear topologies does not exceed 1 in terms of normalized network throughput, or equivalently, channel utilization. We explore transmission schedules in three important contexts: (1) single collision domain with unicast traffic. In an $N$-node network, we develop transmission schedules achieving a normalized network throughput of $2-(2 / N)$. This is the best that can be done in such a context, as demonstrated using a general greedy approach combined with an exhaustive search for small-size networks. (2) single collision domain with broadcast traffic. We propose a periodic per-node fair schedule with the shortest period. Achievable throughput in such conditions is close to $N / 2$. Likewise, we prove that the throughput is upper bounded by $N-1$ under the per-node fairness constraint. (3) partially-overlapping collision domains with unicast traffic. We consider a simple illustration of such a configuration. The proposed transmission schedule depicts a scenario where messages originate at one end of the network, and are sequentially relayed node-by-node (i.e., hop-by-hop) in the direction of the final destination located at the other end of the network. Furthermore, for all three discussed contexts, we build up computationally-efficient algorithms that generate transmission schedules regardless of network size. We explore the idea of exploiting non-zero propagation delays for linear topologies to improve network throughput. In recent UWA sensor networks, the linear topology is a fundamental component that may be used to build more complex network configurations. This study would then serve as a base for future research into this area.
\end{abstract}

\section{INTRODUCTION}

In recent years, underwater acoustic (UWA) sensor network deployments have been explored for use in many applications: offshore survey and exploration, costly infrastructure monitoring, surveillance in sensitive areas and other more general scientific, military and commercial missions. In order to monitor or explore the largest possible area, a network with a linear geometry (wherein the nodes are placed on a single line) may be desirable. As in all 
shared-medium networks, a medium access control (MAC) protocol is necessary, primarily to regulate and coordinate medium access, i.e., how and when nodes access the UWA channel to be able to effectively use this shared medium. Due to the low speed of sound in water (about $1500 \mathrm{~m} / \mathrm{s}$ ), the propagation delays in UWA networks are inherently large, i.e., comparable to or even greater than the packet duration. In an environment with such features, and in view of the linear network architecture, our main objective is to propose transmission schedules that exploit the large propagation delays and make it possible to obtain a desirable level of network throughput, or high channel utilization. This can be achieved by allowing simultaneous transmissions within the network as often as possible, and concentrating interferences at unintended nodes as much as possible. Time division multiple access (TDMA) -based solutions offer more valuable opportunities in this environment, as compared to handshaking protocols and acknowledgment-based retransmission schemes. Furthermore, contention-based MAC protocols are usually more energy-consuming than their TDMA counterparts since they waste energy during collisions and idle listening [1].

In practice, some application scenarios involve unicast delivery within a fully connected zone or equivalently within a single collision domain. A simple example is the sensor network case where each node has to transfer the data it has collected to specific destination nodes. Other applications may bring nodes to share navigation information, command and control system instructions, or even predefined messages for node safety. This requires the use of broadcast traffic. When the extent of the area to be covered gets larger, it becomes necessary to use multihop relaying where a long distance is divided into multiple shorter hops in order to gain more advantageous communication conditions. In fact, the attenuation of the UWA signal depends exponentially on propagation distance, and also on frequency. Thus, bandwidth and power are severely limited [2]. The arrangement of nodes consequently leads to partially-overlapping collision domains.

We consider the scheduling of transmissions between all nodes within a linear $N$-node network in three separate contexts. By exploiting information on propagation delay, we propose throughput-efficient TDMA transmission schedules. The main contributions of this paper are summarized as follows:

Context 1: single collision domain with unicast traffic

- We propose a transmission schedule that achieves a normalized network throughput of $2-(2 / N)$. A greedy search method allows us to achieve this high level of network performance.

- Once the scheduling problem is formulated with respect to throughput, we develop a practical computationallyefficient algorithm that makes it possible to carry out the design of the aforementioned transmission schedule. This algorithm describes an approximate dynamic programming solution that is insensitive to network size.

Context 2: single collision domain with broadcast traffic

- Periodic per-node fair schedule with the shortest period is presented. Achievable throughput in such conditions is demonstrated through a greedy approach. We propose a fast, effective algorithm that allows generation of the transmission schedule.

- As compared to the aforementioned broadcast schedules, we introduce illustrations for multicast schedules with good connectivity and better throughput. Having shorter periods, the proposed schedules perform more 
frequent network transmissions.

- We prove that $N-1$ is the upper bound for the throughput in the case of per-node fair access. Furthermore, we propose per-node fair schedules that may move towards this upper bound as closely as desired, assuming no constraints on the time extent. Likewise, schedules without fairness constraints are also presented.

Context 3: partially-overlapping collision domains with unicast traffic

- Considering a simple illustration of such a configuration, we propose a scheme where messages originating at one end of the network are sequentially relayed node-by-node (i.e., hop-by-hop) in the direction of the final destination located at the other end of the network.

- We present a computationally-efficient algorithm that generates the transmission schedule regardless of network size.

The main idea of taking advantage of large propagation delays has been taken into consideration in many studies. In [3], the authors consider a Request-To-Send(RTS)/Clear-To-Send(CTS)-based protocol for ad hoc networks. This distance-aware protocol makes it possible to increase overall system efficiency by decreasing the average control packet size. In fact, each node uses different control packet sizes for different destination nodes. The gain achieved does not make throughput superior to the optimal performance obtained when the propagation delay is zero (i.e., normalized throughput of 1). An ad hoc handshaking-based scheme was also considered in [4]. Using time information carried by control packets, range between different communicating nodes is estimated. Afterwards, an algorithm employs this delay information for data packet scheduling at each node. According to the node's current schedule, RTS/CTS exchange is used to plan transmission of future data packets. For all the variants studied, this protocol achieves normalized throughput less than 1. The authors in [5] use node position information to overlap communications without collisions in order to increase channel utilization. Likewise, they allow multiple messages to arrive simultaneously at an unintended node. Performance in terms of normalized throughput, as defined by the authors, never reaches 1, irrespective of the network topology adopted. However, if we assume that the final destination node in the third context discussed to be a sink node, our proposed schedule achieves a throughput of 1. The study in [6] essentially highlights the value of adopting bidirectional, concurrent transmissions in UWA networks as regards throughput and end-to-end packet delay. The authors adopt a packet bursting approach, where a senderreceiver pair can exchange multiple rounds of bidirectional packet transmissions. They propose an asynchronous handshaking-based MAC protocol, but because of limiting factors such as idle backoff durations, packet collisions, and control packet exchanges, best performance in terms of the achieved network throughput normalized by the theoretical maximum throughput is again less than 1. Long propagation delay of acoustic signals is also used in [7] to facilitate concurrent transmissions and receptions. In a fully-connected network with one final destination for all nodes, MAC transmission scheduling has been explored for maximum network throughput. Apart from its complexity, the optimal solution found for the model formulated leads to normalized network throughput values that are upper bounded by 1 . The same idea is exploited by the authors in [8] to develop a broadcast transmission schedule for spatial reuse TDMA. The motivation behind this design is still to improve throughput by exploiting 
network topology information. However, the demonstrated performance in terms of packets per time slot does not exceed 1.

A linear topology for multihop networking in UWA systems has been considered to some extent in the literature within the last ten years (e.g. [9]-[12]). Most of the interest therein was on physical link capability. Nevertheless, the authors did not use network-oriented metrics to characterize network performance and find limits. Overlapping transmissions in linear topology also drew interest in the analysis in [2]. Explicitly, in a slotted model for transmissions, each time slot is used by certain nodes to transmit while others receive. With respect to the network model considered therein, traffic originating from one source node at one end is eventually received by a destination node at the other end of the network. Taking into account interhop interference, achievable information rates are optimized over the signal power spectral density, versus per-node power. Nevertheless, no network-oriented performance has been explored. In a UWA sensor network with linear topology, [13] introduces TDMA-based MAC protocol allowing concurrent transmissions in the same time slot. This protocol uses heuristic strategies to improve channel utilization, calculated as the number of transmissions within the network per time unit. Performance evaluation has focused on medium-sized networks. For instance, channel utilization does not exceed $3 / 2$ in the 11-node network. However, in this paper, using the same network structure, we explore transmission schedules with achievable network throughput that may increase proportionally with network size. A multihop linear topology has been studied in [14] under fair access criterion for all nodes. The sensor network model consists of one base station as the final destination of all the network traffic. Sensor nodes are placed along a line with identical distance between every two neighboring nodes. The transmission range for each node is assumed to be just one hop, while the interference range is less than two hops. In this paper, message duration is set to be equal to the one-hop propagation delay for analytical simplicity. With this network model formulation, the authors in [14] proved that under a per-node fair access constraint, an asymptotic upper bound in terms of overall network utilization is $1 / 2$. However, they do not provide transmission schedules that achieve this upper bound. Though we assume the interference range in the third context discussed to be twice the transmission range, we present a 1/2-network utilization (as has been defined in [14]) transmission schedule.

The time interference alignment concept, also referred to as interference alignment by delay, is based on the main idea of overlapping interferences at selected nodes during a given time, and trying to allow the nodes in question to use these periods for transmission. Among the studies that have explored this concept, we cite [15][18]. However, the analysis has been taken a step further by the authors in [19]. They prove that $N / 2$ is an upper bound on the maximum achievable throughput in an $N$-node network with nonzero propagation delay. In addition, they pinpoint some geometries where developed transmission schedules achieve this upper bound. Likewise, they prove the existence of a throughput maximizing optimal schedule that is periodic, for each network. We capitalize on the invaluable findings in [19] to conduct a more sharply focused study. In the context of a linear topology, we carry out an in-depth analysis to explore to what extent we can exploit large propagation delays in consideration of traffic policy, collision domain extent, and certain fairness constraints. We can see that almost all the work cited involves multihop relaying policies within the network. In contrast, we study various scenarios applicable to 
different UWA applications. The three contexts considered provide explicit segmentation of the analysis where we expect to deliver a far deeper understanding and promising results. Note that our focus in this paper is on the MAC sub-layer. Given the regular linear topology, we demonstrate to what extent a TDMA- based MAC protocol can achieve high network throughput in the case of the physical link being reliable.

The remainder of the paper is organized as follows. The general context and system model are described in section II. The first context is widely discussed in section III, which focuses on network throughput in regular linear networks with unicast traffic. In addition, we develop an efficient problem-solving algorithm. Section IV explores achievable throughput with reference to different fairness constraints, in regular linear networks with broadcast traffic. We provide explicit and attainable bounds, and propose a fast algorithm that allows constructing the transmission schedule we suggest first. A representative instance of the third context related to partially-overlapping collision domains with unicast traffic, is depicted in section V. Finally, Section VI concludes the paper.

\section{CONTEXT AND SYSTEM MODEL}

\section{A. Delay matrix}

In a nonzero propagation delay environment, we consider a regular $N$-node linear network where each node is identified by $i$ s.t. $i \in \mathbb{N}^{*}$ and $1 \leq i \leq N$. Let $\mathbf{r}_{i}$ be the position vector of node $i$ in 3-D Euclidean space. The propagation delays between every pair of nodes may be expressed using a delay matrix [19] denoted by $\mathbf{D}$. The time is assumed to be slotted. Thus,

$$
D_{i j}=\frac{\left|\mathbf{r}_{i}-\mathbf{r}_{j}\right|}{c \tau}, \quad 1 \leq i, j \leq N
$$

where $c$ is the signal propagation speed and $\tau$ is the length of one time slot. Hence, the entries in the delay matrix are nonnegative real numbers. Furthermore, we consider in this paper a regular $N$-node linear network with unit spacing between every pair of neighboring nodes. Indeed, for the sake of analytical simplicity, we have also assumed that the time slot duration is set to the propagation delay between two neighboring nodes. As shown next, there is no loss of generality in this assumption. Provided that we seek to improve channel utilization, in the case where slots are an integer fraction $1 / K(K>1)$ of unit propagation delay, one can for instance consider an arbitrary transmission cycle where every node $i$ transmits $m_{i}$ times over the duration of one cycle $T_{\text {cycle }}$ (which is not necessarily a multiple of $K$ ). By repeating the cycle $K$ times, we end up with a transmission schedule having a time extent of $\Pi=K T_{\text {cycle }}$, during which every node $i$ transmits $m_{i} K$ times. This can be seen as node $i$ transmitting during $m_{i}$ units propagation delay. To make things simpler, an illustration where all slots are well occupied consists in transmitting $K$ successive messages for each node during one unit propagation delay. This could be reduced to transmit one message of a duration equal to one unit propagation delay, as assumed in our model for the sake of analytical simplicity, while one may, furthermore, make gains on overheads of $K$ messages. Therefore, no additional insight is provided by considering the general case with slots that are an integer fraction of unit propagation delay in a linear network with regularity in the distances separating nodes. The delay matrix 
entries are then natural numbers and $D_{i j}$ becomes

$$
D_{i j}=|i-j|, \quad \forall i, j
$$

The linear geometry of the network under consideration is fully described by the delay matrix. Besides, $\mathbf{D}$ is symmetric, i.e., $D_{i j}=D_{j i}$, since $|i-j|=|j-i|$, and has an all-zero diagonal, i.e., $D_{i i}=0($ since $|i-i|=0)$. The delay matrix corresponding to the 3-node regular linear network is given by $\mathbf{D}_{3}$.

$$
\mathbf{D}_{3}=\left[\begin{array}{lll}
0 & 1 & 2 \\
1 & 0 & 1 \\
2 & 1 & 0
\end{array}\right]
$$

\section{B. Network environment features}

All nodes in the network are operating in half-duplex mode, i.e., a node cannot simultaneously transmit and receive. In a single collision domain, or equivalently a fully connected network, a message transmitted by a node reaches all other nodes in the network after the corresponding propagation delays. With reference to the traffic type, unicast transmission identifies with a message sent from a single source node to a single destination node, while multicast transmission is used to refer to a message sent from a single source node to a set of destination nodes within the network. If this set of destination nodes includes all nodes other than the transmitting node, we deal with broadcast traffic.

In this paper, we consider three scenarios of interest: single collision domain with unicast traffic, single collision domain with broadcast traffic, and a simple illustration of partially-overlapping collision domains with unicast traffic. Environment monitoring and tactical surveillance in a specific zone, as well as many other applications, are carried out through underwater sensor networks where the first and the second scenarios are quite appropriate. In view of the numerous communication link challenges in a doubly selective underwater acoustic channel, the transmit power for each node is generally high enough to reach the whole network. If the extent of the monitored area becomes wider, the third scenario is more pertinent.

In the case of the first scenario, excluding the source and destination nodes, a message is considered as an interference at all nodes it reaches. On the other hand, in the second scenario, a message is considered to be an interference at a node only if it overlaps in time with another message intended for that node. However, in the context of the third scenario, it becomes necessary to cope with two types of interference constraints, both related to the strength of the detected signal, i.e., whether it is high enough to decode or not. A message is not only an interference at all nodes between the source and destination nodes within the collision domain in question. It is also an interference at the surrounding nodes, where the signal is not sufficiently strong to be decoded, while it is still detectable.

\section{Throughput and transmission schedule}

Assuming the physical link to be reliable (error-free) with constant data rate $\nu$, the loss of a message is only due to collision. A collision is said to occur at a certain node if two or more messages overlap in time, while a 
successful transmission is defined as a transmission that results in a successful reception (or receptions when it comes to multicast traffic) of the message at the destination node (or nodes). The normalized network throughput (also designated by network throughput or simply throughput) $Y$ is the total number of information bits successfully received by all nodes in the network per unit time, normalized by the link data rate $\nu$.

Provided that the message duration is equal to $\tau$, which is also the propagation delay between two adjacent nodes, we define a transmission schedule (or simply schedule) $\mathbf{S}$ as the matrix that determines when each node transmits and receives messages [19]. As regards the entries of $\mathbf{S}$

- $S_{i t}=S_{i, t}=j>0$ indicates that node $i$ transmits a message to node $j$ at time slot $t$

- equivalently, in the case of multicast delivery, $S_{i t}=S_{i, t}=j k l>0$ signifies that node $i$ transmits a message to nodes $j, k$ and $l$ at time slot $t$

- $S_{j t}=S_{j, t}=-i<0$ implies that node $j$ receives a message from node $i$ during the time slot $t$

- in all other cases, node $i$ is designated as an idle node during time slot $t$, which is represented by $S_{i t}=S_{i, t}=0$

If $S_{i, t+T}=S_{i t}, \forall i, t$, this indicates that the transmission schedule is repeating with a period $T$. It is said to be periodic with period $T$ and may be depicted using an $N \times T$ matrix $\mathbf{S}^{(T)}$ where

$$
S_{i t}=S_{i, t(\bmod T)}^{(T)}
$$

By way of illustration, we might consider the following transmission schedule in the 3-node network with unicast traffic

$$
\mathbf{S}^{(3)}=\left[\begin{array}{ccc}
3 & 2 & -3 \\
3 & 0 & -1 \\
1 & -2 & -1
\end{array}\right]
$$

A simple explanation of the transmissions and the corresponding receptions handled in this schedule is reported in Table I. If the columns of matrix $\mathbf{S}^{(3)}$ are circularly shifted to the right or to the left, the resulting matrix describes

TABLE I

SUMMARY OF PLANNED TRANSMISSIONS AND CORRESPONDING RECEPTIONS IN ACCORDANCE WITH $\mathbf{S}^{(3)}$

\begin{tabular}{|c|c|c|c|}
\hline \hline Node $\backslash$ time slot & time slot $\mathbf{1}$ & time slot $\mathbf{2}$ & time slot 3 \\
\hline Node $\mathbf{1}$ & Transmission to node 3 & Transmission to node 2 & Reception from node 3 \\
\hline Node $\mathbf{2}$ & Transmission to node 3 & Idle & Reception from node 1 \\
\hline Node 3 & Transmission to node 1 & Reception from node 2 & Reception from node 1 \\
\hline
\end{tabular}

the same transmission schedule.

From schedule $\mathbf{S}^{(T)}$ with period $T$, the average network throughput can be calculated considering the number of receptions in schedule $\mathbf{S}^{(T)}$

$$
Y=\frac{1}{T} \sum_{t=0}^{T-1} \sum_{i=1}^{N} \mathbb{1}\left(S_{i t}^{(T)}<0\right)
$$

where $\mathbb{1}(E)$ is the indicator function of event $E$, with value 1 if $E$ is true and 0 otherwise. 


\section{Fair schedule types}

The set of different access opportunities between nodes should be taken into consideration, such that the access during one period $T$ is done in a certain fair way. The objective is always to ensure a high network utilization, i.e., a high throughput. We use the terminology of fairness as introduced in [19].

When a schedule $\mathbf{S}^{(T)}$ provides the same number of transmission opportunities to all nodes, $\mathbf{S}^{(T)}$ is said to be per-node fair. This signifies that

$$
\sum_{t=0}^{T-1} \mathbb{1}\left(S_{i t}^{(T)}>0\right)=\text { constant }>0, \quad \forall i
$$

However, with respect to unicast traffic, we can add the stronger fairness characteristic of transmitting to all other nodes at the same rate. This allows us to refer to $\mathbf{S}^{(T)}$ as a per-link fair schedule. Correspondingly,

$$
\sum_{t=0}^{T-1} \mathbb{1}\left(S_{i t}^{(T)}=j\right)=\text { constant }>0, \quad \forall i, j \neq i
$$

In spite of that, we may obtain a lower level of fairness by offering to all nodes the opportunity to transmit to all other nodes at least $m>0$ times during one period $T$, which would imply

$$
\sum_{t=0}^{T-1} \mathbb{1}\left(S_{i t}^{(T)}=j\right) \geq m, \quad \forall i, j \neq i
$$

Such a transmission scheme might be referred to as a weakly per-link fair schedule. Likewise, a weakly per-node fair schedule can be used to describe the case where all nodes have the opportunity to transmit at least $n>0$ times during one period $T$, i.e.,

$$
\sum_{t=0}^{T-1} \mathbb{1}\left(S_{i t}^{(T)}>0\right) \geq n, \quad \forall i
$$

A fairness criterion as has been introduced will be one of the main components considered in developing algorithms that generate transmission schedules of interest.

\section{THROUGHPUT IN A REGULAR LINEAR NETWORK WITH A SINGLE COLLISION DOMAIN AND UNICAST} TRAFFIC

\section{A. Achievable network throughput and illustrative transmission schedules}

1) Achievable network throughput: Let us first clarify what is meant by a useful message. Once transmitted from a source node $i$ to a destination node $j$, the message is considered to be useful while it does not reach yet the destination, i.e., certainly it is an interference (positive interference) on all nodes that are in between $i$ and $j$, but it carries useful information intended for $j$, unlike its zero utility (negative interference) at all the other nodes outside the segment $i \leftrightarrow j$, where it is rather a useless message. In Fig. 1, an effective useful message is referred to as a confirmed useful message.

In a transmission schedule matrix under development, an entry is said to be advantageous if it can be subject to two overlapping useful messages traveling in opposite directions. However, an entry is said to be restricted if there 
is already a useless message (negative interference) such that it can accommodate a maximum of one single useful message.

Lemma 1. In a regular $N$-node linear network with a single collision domain and unicast traffic, a transmission from node $i$ to node $j$ at time slot $t$ generates $2\left(N-1-D_{i j}\right)$ restricted entries in a transmission schedule matrix under development, along with $D_{i j}-1$ advantageous entries.

Proof: Node $i$ transmits a message to node $j$ during time slot $t$ only if node $j$ is able to successfully receive the message during time slot $t+D_{i j}$. Therefore,

$$
S_{i t}=j \quad \Leftrightarrow \quad S_{j, t+D_{i j}}=-i \quad \forall i \neq j
$$

Furthermore, in order to ensure the successful reception of a transmitted message at time slot $t$, it is required that no other nodes transmit messages that arrive at node $j$ during time slot $t$. Thus,

$$
S_{j t}=-i \quad \Rightarrow \quad S_{k, t-D_{j k}} \leq 0, \forall k \neq i
$$

Let us assume that $i<j$.

First category of restrictions generated:

Once emitted at $t$, the message of node $i$ will transit within the network in both directions. Then, it is a negative interference (useless message) on each node $k, 1 \leq k<i$ at future time slot $t+i-k$. Hence, $i-1$ nodes will be affected, due to this transmission, after $t$. In terms of the transmission schedule matrix under development, each of the corresponding $i-1$ entries is impacted by one useless message, and the best that can be done to increase the network throughput is to allow the transit of a useful message (once it is possible). In a similar way, after reaching node $j$ at $t+D_{i j}$ (according to (11)), the message sent by node $i$ is a negative interference (useless message) on each node $l, j<l \leq N$ at future time slot $t+D_{i j}+l-j$. Thus, this negative interference created by the transmission in question will get at $N-j$ additional nodes. In the transmission schedule matrix under development, each of $N-j$ related entries is inevitably impacted, and it would be desirable to be able to transmit. The total number of resulting restrictions due to useless message is $N-j+i-1=N-D_{i j}-1$, since $D_{i j}=j-i$. A graphical illustration of this first category of restrictions is given in Fig. 1, where the second category is also depicted.

Second category of restrictions generated:

Furthermore, let us focus on time slots before $t$. Based on (12), each node $k, 1 \leq k<i$ should not be transmitting at previous time slot $t-i+k$. In the best-case scenario, one would then expect the reception (or the transit) of a useful message at the corresponding entry. This concerns $i-1$ entries in the transmission schedule matrix. Additionally, the same constraint is applied to each node $l, j<l \leq N$ at time slot $t+D_{i j}-l+j$, before the scheduled time slot for reception, i.e., $t+D_{i j} . N-j$ supplementary entries are implied. Thus, the second category of restrictions involves $N-j+i-1=N-D_{i j}-1$ entries in the transmission schedule matrix.

It should be noted that each of the total $2\left(N-D_{i j}-1\right)$ entries subject to restrictions may accommodate a maximum of one useful message, being transmitted or transiting. In contrast, each of the intermediate entries 


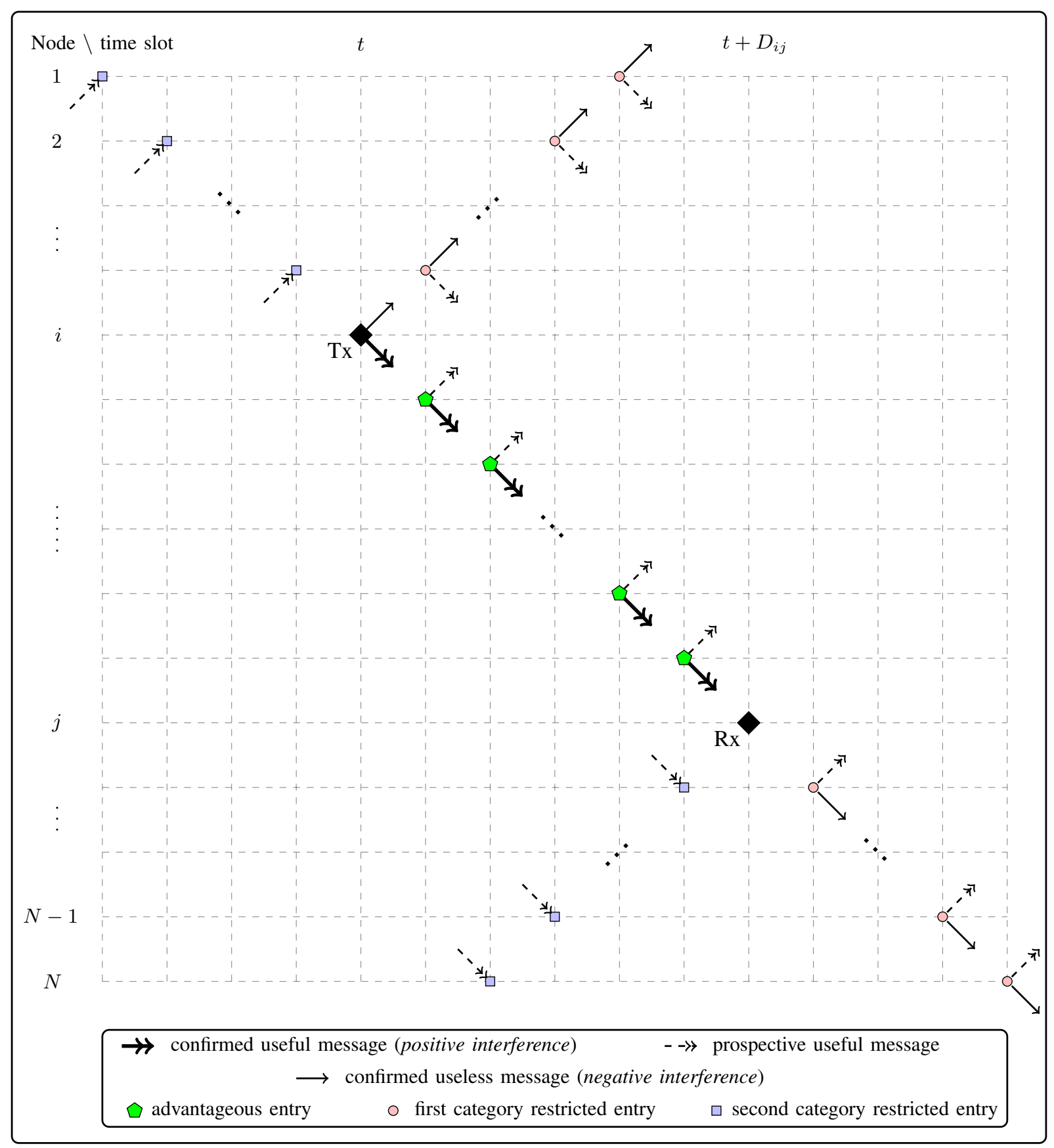

Fig. 1. Depiction of the network state when node $i$ transmits to node $j(i<j)$ 
corresponding to $j-i-1=D_{i j}-1$ intermediate nodes $m, i<m<j$, is idle at time slot $t+m-i$ with one useful message in transit. However, it can potentially accommodate a second useful message as described in Fig. 1, as long as the ultimate goal is to increase the network throughput.

In sum, transmission from $i$ to $j$ generates $2\left(N-D_{i j}-1\right)$ restricted entries in the transmission schedule matrix, in addition to $D_{i j}-1$ advantageous entries.

The result is the same when $i>j$.

Theorem 1. In a regular $N$-node linear network with a single collision domain and unicast traffic, the maximum achievable throughput is $2-(2 / N)$.

Proof: The general forms of schedules for $N$ even and $N$ odd are summarized as $\mathbf{S}^{(N)}$ an $\underline{\mathbf{S}}^{(N)}$ respectively. However, let us first show how we built these solutions. Given the linear geometry, the network can be considered as a set of $N-1$ adjacent sections. Each section consists of two neighboring nodes. Let us use exchange-state to denote the activity within the network in terms of messages being transmitted, received or still circulating in the network. At a certain time slot, the network exchange-state that hosts the maximum traffic of useful messages is the exchange-state where each section accommodates two messages simultaneously. It obviously comes to two useful messages transiting in opposite directions, or equivalently one message transiting and the other being received, but still with two flying useful messages on each of the two surrounding sections (if they exist). Actually, this last case concerns only the two sections at the ends of the network. Let Net opt denote this optimum exchange-state.

While seeking to maximize the network throughput, the transmission schedule should ensure that $N e t_{o p t}$ is reached as quickly as possible, and then allow the receptions to likewise be performed as quickly as possible. $2(N-1)$ is the number of messages corresponding to $N e t_{o p t}$, and since the network includes only $N$ nodes, this state cannot be reached after one time slot. It should be achieved gradually. Let us grant access to all nodes of the network during the first time slot $t$. In doing so, we will have $N$ useful messages in the network. However, according to Lemma 1 , any transmission from node $i$ to node $j$ results in $2\left(N-D_{i j}-1\right)$ restricted entries in the transmission schedule matrix, as well as $D_{i j}-1$ advantageous entries. Therefore, three key elements should be taken into account. Firstly, for each transmission, selected $D_{i j}$ should be as high as possible in order to minimize negative interference and maximize potential upcoming transmissions. Secondly, generated negative interferences are to be managed in such a way that they leave the network as rapidly as possible, and ideally, are replaced by useful messages. Lastly, consider condensing receptions to take the shortest possible time.

Let us consider an $N$-node network where $N$ is even.

At time slot 1 , for each node $i, 1 \leq i \leq(N / 2)$, we select node $N$ as the $j$ destination in order to maximize the distance $D_{i j}$. As regards nodes $k$ where $(N / 2)+1 \leq k \leq N$, they have node 1 as their destination. Thus far, we have at $1,(N / 2)$ contiguous useful transmissions on the right half of the network, oriented to the center, as well as $N / 2$ other contiguous useful transmissions on the left half. $N$ simultaneous transmissions $((N / 2)$ in one direction and $(N / 2)$ in the other) give rise to $N-2$ negative interferences, since the nodes at the edges do not generate 
negative interference, while the others generate one negative interference each. However, it should be noted that the $((N-2) / 2)$ negative interferences on the right half of the network are all oriented to the right end (i.e., extremity node $N$ ), which is the nearest end. In parallel, the situation is the same on the left half, with negative interferences pointing to the left end (i.e., extremity node 1). Therefore, all negative interferences will, in pairs, completely clear out the network at $(N / 2)$. One pair evacuating at each running time slot $1+e, 1 \leq e \leq(N / 2)-1$ is the maximum we can achieve in terms of rapid negative interference evacuation. Nevertheless, it is important to replace each evacuating negative interference by a new useful message as long as we aim to reach $N e t_{\text {opt }}$. To do so, we need to look for transmissions that do not introduce additional negative interference, and try to schedule the corresponding receptions within a time horizon not exceeding the last time slot planned for the reception of previous transmissions. Only nodes 1 and $N$ can transmit without causing negative interference. Thus, the following transmissions follow the general form of the schedule summarized in (13) as $\mathbf{S}^{(N)}$.

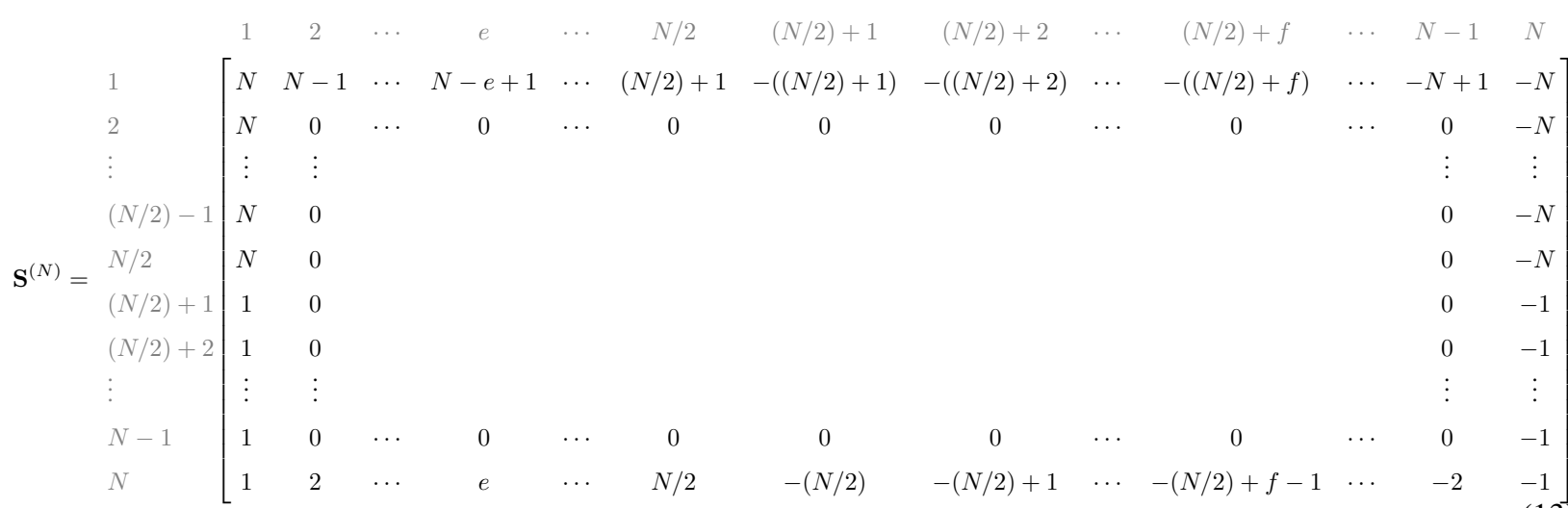

In the case where $N$ is odd, the proposed transmission scheme is slightly modified compared to that for $N$ even. The changes are identified in the general form of schedule $\underline{\mathbf{S}}^{(N)} \cdot \mathbf{S}^{(N)}$ and $\underline{\mathbf{S}}^{(N)}$ are weakly per-link fair, since each node transmits at least once during one period $N$.

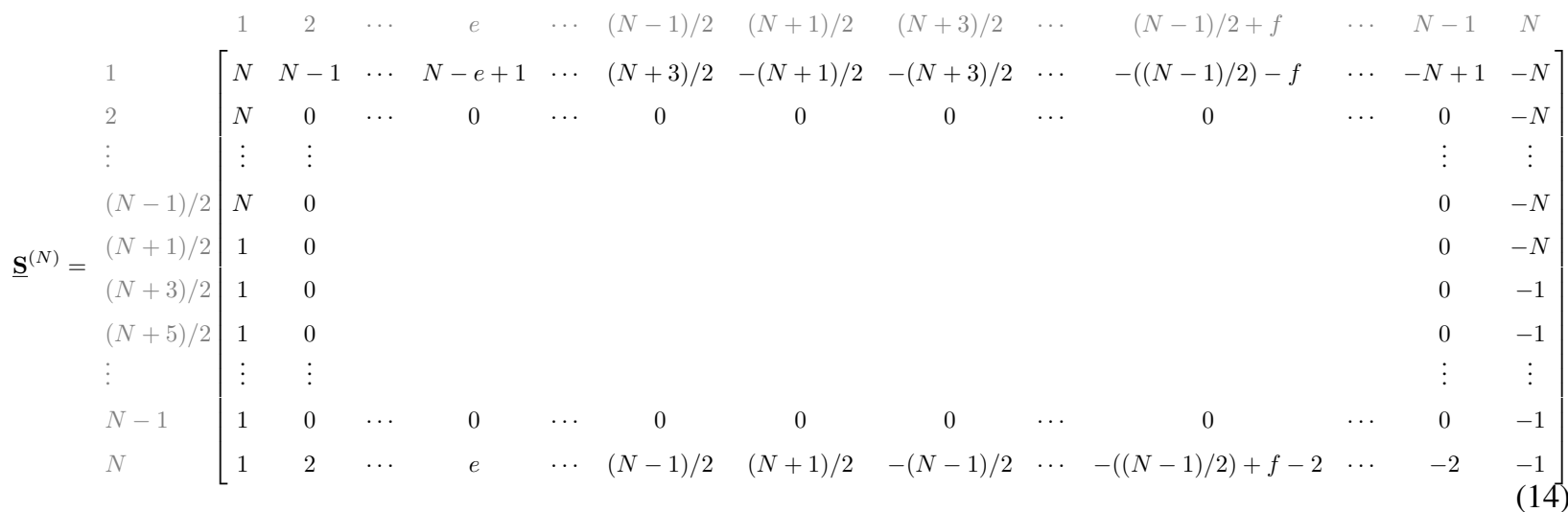

As we are interested in the presence of useful messages in the network and their receptions, let us follow up the evolution of the transmissions until the network reaches $N_{\text {ept }}$, and then the receptions until all messages reach their respective destinations. Table II describes the progress of this process for $N$ even.

From $\mathbf{S}^{(N)}$ and $\underline{\mathbf{S}}^{(N)}$, we notice that the period duration is $N$ and the number of transmissions within one 
TABLE II

OVERVIEW OF THE NETWORK exchange-state EVOLUTION OVER TIME ( $N$ EVEN)

\begin{tabular}{|c|c|c|}
\hline \hline Time slot & $\begin{array}{c}\text { Number of useful messages } \\
\text { being transmitted, received or } \\
\text { still circulating in the network }\end{array}$ & Notes \\
\hline$t$ & $N$ & $N$ simultaneous transmissions \\
\hline$t+1$ & $N+2$ & 2 additional transmissions \\
\hline$\ldots$ & $N+2 e$ & idem \\
\hline$t+e$ & $2(N-1)$ & first 2 receptions \\
\hline$\cdots$ & $2(N-1)$ & (no more transmissions) \\
\hline$t+(N / 2)-1$ & & 2 additional receptions \\
\hline$t+(N / 2)$ & $2(N-1)-2$ & idem \\
\hline$t+(N / 2)+1$ & & idem \\
\hline$\cdots$ & $2(N-1)+N-2 f$ & $N$ receptions \\
\hline$t+f$ & & \\
\hline$\cdots$ & $N+2$ & last \\
\hline$t+N-2$ & $N$ & \\
\hline$t+N-1$ & & \\
\hline
\end{tabular}

period is $N+2((N / 2)-1)=2(N-1)$. Hence, the corresponding network throughput in such an $N$-node linear network is $2-2 / N$. It is also worth pointing out that, for validation and strengthening purposes, we performed the exhaustive search over small-size networks ( $3 \leq N \leq 5)$, although the time needed is prohibitively long. We found that $2-2 / N$ is the best that can be done in terms of the normalized network throughput.

2) Illustrative transmission schemes: In order to have a clear view of the proposed transmission schedules, we provide illustrative examples. For instance, let us consider 4-node and 5-node regular linear networks. The corresponding schedules are represented by $\mathbf{S}^{(4)}$ and $\mathbf{S}^{(5)}$ respectively.

$$
\begin{gathered}
\mathbf{S}^{(4)}=\left[\begin{array}{cccc}
4 & 3 & -3 & -4 \\
4 & 0 & 0 & -4 \\
1 & 0 & 0 & -1 \\
1 & 2 & -2 & -1
\end{array}\right] \\
\mathbf{S}^{(5)}=\left[\begin{array}{lllll}
5 & 4 & -3 & -4 & -5 \\
5 & 0 & 0 & 0 & -5 \\
1 & 0 & 0 & 0 & -5 \\
1 & 0 & 0 & 0 & -1 \\
1 & 2 & 3 & -2 & -1
\end{array}\right]
\end{gathered}
$$

It is worth mentioning that the proposed transmission schedule, achieving $2-(2 / N)$ network throughput, is not unique. Several schedules reaching the same performance can be found, especially for high values of $N$. To put 
it in context, let us examine the example of 7 -node network. According to the approach discussed above, the corresponding transmission schedule is depicted by $\mathbf{S}_{<1>}^{(7)}$. The index $<1>$ is used to denote the first alternative derived.

$$
\mathbf{S}_{<1>}^{(7)}=\left[\begin{array}{ccccccc}
7 & 6 & 5 & -4 & -5 & -6 & -7 \\
7 & 0 & 0 & 0 & 0 & 0 & -7 \\
7 & 0 & 0 & 0 & 0 & 0 & -7 \\
1 & 0 & 0 & 0 & 0 & 0 & -7 \\
1 & 0 & 0 & 0 & 0 & 0 & -1 \\
1 & 0 & 0 & 0 & 0 & 0 & -1 \\
1 & 2 & 3 & 4 & -3 & -2 & -1
\end{array}\right]
$$

With minor modifications we can come up with $\mathbf{S}_{<2>}^{(7)}$ and $\mathbf{S}_{<3>}^{(7)}$ also performing a network throughput of (12/7). Actually, the changes made in terms of receptions affect idle entries in the transmission schedule matrix that are impacted by a single interference (as opposed to at least two interferences in opposite directions).

$$
\mathbf{S}_{<2>}^{(7)}=\left[\begin{array}{ccccccc}
7 & 6 & 5 & -4 & -5 & 0 & 0 \\
7 & 0 & 0 & 0 & -6 & -7 & -7 \\
7 & 0 & 0 & 0 & 0 & 0 & -7 \\
1 & 0 & 0 & 0 & 0 & 0 & -7 \\
1 & 0 & 0 & 0 & 0 & 0 & -1 \\
2 & 0 & 0 & 0 & 0 & 0 & -1 \\
2 & 2 & 3 & 4 & -3 & -2 & -1
\end{array}\right] \quad \mathbf{S}_{<3>}^{(7)}=\left[\begin{array}{ccccccc}
6 & 6 & 5 & -4 & -5 & -6 & -7 \\
7 & 0 & 0 & 0 & 0 & 0 & 0 \\
7 & 0 & 0 & 0 & 0 & -7 & -7 \\
1 & 0 & 0 & 0 & 0 & 0 & -7 \\
1 & 0 & 0 & 0 & 0 & 0 & -1 \\
1 & 0 & 0 & 0 & 0 & -1 & -1 \\
1 & 3 & 3 & 4 & -3 & -2 & 0
\end{array}\right]
$$

Moreover, unlike $N$-node networks with $N$ even, the proposed transmission schedule for $N$ odd is unbalanced. Specifically, over $N$ time slots (one period), node 1 transmits $((N-1) / 2)$ times and is involved as destination node $((N+1) / 2)$ times at the starting time slot. Node $N$ transmits $((N+1) / 2)$ times concurrently while it is intended $((N-1) / 2)$ times. Hence, a simple permutation provides a new schedule. After replacing 1 in row 4 column 1 of $\mathbf{S}_{<1>}^{(7)}$ by 7 , and -4 in row 1 column 4 by 4 , and considering the corresponding receptions, we obtain $\mathbf{S}_{<4>}^{(7)}$. If such a permutation is combined with the aforementioned modifications, it will result in many new transmission schedules with the same network throughput.

$$
\mathbf{S}_{<4>}^{(7)}=\left[\begin{array}{ccccccc}
7 & 6 & 5 & 4 & -5 & -6 & -7 \\
7 & 0 & 0 & 0 & 0 & 0 & -7 \\
7 & 0 & 0 & 0 & 0 & 0 & -7 \\
7 & 0 & 0 & 0 & 0 & 0 & -1 \\
1 & 0 & 0 & 0 & 0 & 0 & -1 \\
1 & 0 & 0 & 0 & 0 & 0 & -1 \\
1 & 2 & 3 & -4 & -3 & -2 & -1
\end{array}\right]
$$




\section{B. Problem modeling and solution algorithm}

1) Problem modeling: The geometry of an $N$-node network is depicted by the delay matrix $\mathbf{D}$ and the size of the network is given by

$$
A=\max _{i, j} \mathbf{D}_{i j}
$$

In the case of a regular $N$-node linear network, the corresponding size is $A=N-1$.

As in [19], we formulate the problem of finding an optimal schedule, denoted by $\mathbf{S}^{*}$, as a sequential decision problem. Since every network has an optimal schedule that is periodic [19], we are interested in schedule $\mathbf{S}^{*}$ with period $T$. The current state of the system is known and represented by $\mathbf{S}^{\{t\}}$, which is the partial schedule given all transmissions occurring between time slots $t-A$ and $t-1$. It should be remembered here that a transmission will not remain in the network more than $A$ time slots. Indeed, $\mathbf{S}^{\{t\}}$ contains all transmissions made at $t-1$ and $A-1$ time slots earlier. However, resulting receptions and interference at later time slots are taken into consideration in the current state $\mathbf{S}^{\{t\}}$. Let $\mathcal{S}$ denote the discrete state space of $\mathbf{S}^{\{t\}}$. Node $i$ is said to be available at time slot $t$, if $i$ is neither the intended recipient nor subject to paralyzing interference. By paralyzing interference, we mean two or more unintended messages in opposite directions, which impede the node from transmitting. At time slot $t$, the decision $x_{i}^{\{t\}}$ to be taken at each available node $i$ involves granting access to $i$, i.e., $x_{i}^{\{t\}}>0$, or maintaining $i$ idle, i.e., $x_{i}^{\{t\}}=0$. Let $\mathcal{X}\left(\mathbf{S}^{\{t\}}\right)$ denote the set of all possible decisions (also referred to as actions), whereas $\mathcal{X}$ designates the decision space. The effect of action $\mathbf{x}^{\{t\}}=\left(x_{1}^{\{t\}}, x_{2}^{\{t\}}, \cdots, x_{N}^{\{t\}}\right)$ is defined in the state transition equation (21), which produces the new state $\mathbf{S}^{\{t+1\}}$.

$$
\mathbf{S}^{\{t+1\}}=\Delta\left(\mathbf{S}^{\{t\}}, \mathbf{x}^{\{t\}}\right)
$$

In other words, with the transition function $\Delta($.$) using transmissions in \mathbf{x}^{\{t\}}$, the current state $\mathbf{S}^{\{t\}}$ is updated to the next state $\mathbf{S}^{\{t+1\}}$. The transition reward is nothing other than the number of transmissions introduced by action $\mathbf{x}^{\{t\}}$. It can be expressed as

$$
H\left(\mathbf{x}^{\{t\}}\right)=\sum_{i=1}^{N} \mathbb{1}\left(x_{i}^{\{t\}}>0\right), \quad \forall \mathbf{x}^{\{t\}} \in \mathcal{X}\left(\mathbf{S}^{\{t\}}\right)
$$

By taking optimal actions $\left(\mathbf{x}^{*\left\{t^{\prime}\right\}}, \mathbf{x}^{*\left\{t^{\prime}+1\right\}}, \ldots, \mathbf{x}^{*}\left\{t^{\prime}+T-1\right\}\right)$, we come up with an optimal schedule $\mathbf{S}^{*}$. This optimal strategy $X^{*}$, where

$$
\mathbf{x}^{*\left\{t^{\prime}\right\}}=X^{*}\left(\mathbf{S}^{\left\{t^{\prime}\right\}}\right)
$$

provides the maximum throughput designated by $Y^{*}$. Thus, using the transition rewards over one period $T$, the value of $Y^{*}$ is computed by

$$
Y^{*}=\frac{1}{T} \sum_{t^{\prime}=1}^{T} H\left(\mathbf{x}^{*\left\{t^{\prime}\right\}}\right)
$$

Note that there may be more than one optimal strategy. In terms of the dynamic programming approach adopted in [19], $Y(\mathbf{S}, \mathbf{x}):(\mathcal{S}, \mathcal{X}) \longrightarrow \mathbb{R}_{+}$can be seen as the objective function having $Y^{*}$ as the best possible value. 
Besides, the action value function denoted by $Q(\mathbf{S}, \mathbf{x}):(\mathcal{S}, \mathcal{X}) \longrightarrow \mathbb{R}_{+}$, can be used to describe the optimal strategy $X^{*}$

$$
X^{*}(\mathbf{S})=\underset{\mathbf{x} \in \mathcal{X}(\mathbf{S})}{\arg } \max \left(H(\mathbf{x})+Q\left(\Delta(\mathbf{S}, \mathbf{x}), \mathbf{x}^{\prime}\right)\right),
$$

where $\mathbf{x}^{\prime}$ is the decision immediately following $\mathbf{x}$. It will be recalled that satisfying the Bellman equation, the action value function can be expressed in its recursive form as

$$
Q(\mathbf{S}, \mathbf{x})=\max _{\mathbf{x} \in \mathcal{X}(\mathbf{S})}\left(H(\mathbf{x})+Q\left(\Delta(\mathbf{S}, \mathbf{x}), \mathbf{x}^{\prime}\right)\right)
$$

Since we do not know the true action value function, it should be estimated iteratively at each time slot. To this end, standard algorithms require performing exhaustive state space and decision space enumerations. Thus, the complexity grows very fast with the size of the network. The approximation of the action value function is a more practical technique. Although it is a sub-optimal solution, it surprisingly achieves good performance. This method relies on the concept of approximate dynamic programming [20].

2) Practical algorithm: Instead of using decision space $\mathcal{X}$ having a cardinality of $\mathcal{O}\left(N^{N}\right)$, we use successive sequential transmission decisions [19] within one time slot. Each decision is represented by a 2-tuple $(k, l)$ for a single transmission from node $k$ to node $l$ at $t$. Thus, the computational complexity of the decision space enumeration problem is reduced to $\mathcal{O}\left(N^{3}\right)$. We will therefore introduce a new numbering scale, designated by $a$, within each time slot $t$. Let $H^{\{t\}}$ denote the number of transmissions in time slot $t$. After $a-1$ transmission decisions and using the transition function $\Delta($.$) , the partial schedule \dot{\mathbf{S}}^{\{t, a\}} \in \dot{\mathcal{S}}$ is combined with the transmission decision $\dot{\mathbf{x}}^{\{t, a\}}$ to find the next partial schedule, as indicated by

$$
\begin{aligned}
& \dot{\mathbf{S}}^{\{t, a+1\}}=\Delta\left(\dot{\mathbf{S}}^{\{t, a\}}, \dot{\mathbf{x}}^{\{t, a\}}\right), \quad \forall a<H^{\{t\}} \\
& \dot{\mathbf{S}}^{\{t+1,1\}}=\Delta\left(\dot{\mathbf{S}}^{\left\{t, H^{\{t\}}\right\}}, \dot{\mathbf{x}}^{\left\{t, H^{\{t\}}\right\}}\right)
\end{aligned}
$$

In agreement with the new formulation of the problem within state space $\dot{\mathcal{S}}$ and decision space $\dot{\mathcal{X}}$, we introduce an action value function

$$
\begin{aligned}
\dot{Q}(\dot{\mathbf{S}}, \dot{\mathbf{x}}) & =\max _{\dot{\mathbf{x}} \in \dot{\mathcal{X}}(\dot{\mathbf{S}})}\left(H(\dot{\mathbf{x}})+\dot{Q}\left(\Delta(\dot{\mathbf{S}}, \dot{\mathbf{x}}), \dot{\mathbf{x}}^{\prime}\right)\right), \\
& =\max _{\dot{\mathbf{x}} \in \dot{\mathcal{X}}(\dot{\mathbf{S}})} \dot{Q}\left(\Delta(\dot{\mathbf{S}}, \dot{\mathbf{x}}), \dot{\mathbf{x}}^{\prime}\right)+1,
\end{aligned}
$$

since $H(\dot{\mathbf{x}})=1$ is the reward for the single transmission decided within the considered time slot. Note that at the stage illustrated by (29), only decision $\dot{\mathbf{x}}$ matters in the optimal action finding process, unlike $\dot{\mathbf{x}}^{\prime}$. As stated above, the true value of $\dot{Q}$ may be obtained using computationally expensive techniques such as relative value iteration. Nevertheless, their complexity makes them quickly infeasible, especially for networks for a significant size $A$. As a practical and much simpler alternative, a good approximation of the value function can be developed on the basis of our apprehension of the problem structure and properties.

The key feature for any solution is the component current statelfuture states that should evolve towards maximizing the throughput (i.e., maximizing transmissions). We have seen that the time extent of the current state is $A$. Similarly, 
any upcoming transmission will act on the network within $A$ time slots, and we seek to maximize the number of future transmissions. Therefore, one must go through a state that allows this. Given a transmission decision $\dot{\mathrm{x}}$, the capacity of a state to accommodate future transmissions within $A$ time slots is used thus far to measure the state-action pair value, denoted by $\vartheta_{i}\left(\dot{\mathbf{S}}^{\{t\}}, \dot{\mathbf{x}}\right)$.

Let us first enumerate the constraints related to an $N$-node network in the UWA environment. Knowing the partial schedule $\dot{\mathbf{S}}^{\{t\}}$ at $t$, a single transmission from node $k$ to node $l$ at $t^{\prime} \geq t$ is allowed only if

- it is not a self-transmission, i.e., $l \neq k$

- there is no transmission or reception already planned for node $k$ at $t^{\prime}$, i.e., $\dot{S}_{k, t^{\prime}}^{\{t\}}=0$

- there is no transmission or reception already planned for node $l$ at $t^{\prime}+D_{k l}$, i.e., $\dot{S}_{l, t^{\prime}+D_{k l}}^{\{t\}}=0$

- there is no interference at node $l$ at $t^{\prime}+D_{k l}$, which originates from any other node $i(i \neq k)$ at $t^{\prime}+D_{k l}-D_{i l}$, i.e., $\nexists i$ s.t. $\dot{S}_{i, t^{\prime}+D_{k l}-D_{i l}}^{\{t\}}>0$

- the transmission from node $k$ will not cause interference on any other node $j(j \neq l)$ at $t^{\prime}+D_{k j}$, while $j$ is receiving a message from node $i$, i.e., $\nexists i, j$ s.t. $\dot{S}_{i, t^{\prime}+D_{k j}-D_{i j}}^{\{t\}}=j$

In order to summarize this first category of constraints, let us use the transmission indicator function $C_{k l \delta}$ where $\delta=t^{\prime}-t$, as introduced in [19]. The action value function approximation should take into account $C_{k l \delta}\left(\dot{\mathbf{S}}^{\{t\}}\right)$, which is given by

$$
C_{k l \delta}\left(\dot{\mathbf{S}}^{\{t\}}\right)=\left\{\begin{array}{lll}
0, & \text { if } \quad l=k \\
0, & \text { if } \quad \dot{S}_{k, t^{\prime}}^{\{t\}} \neq 0 \\
0, & \text { if } \quad \dot{S}_{l, t^{\prime}+D_{k l}}^{\{t\}} \neq 0 \\
0, & \text { if } \quad \exists i \text { s.t. } \dot{S}_{i, t^{\prime}+D_{k l}-D_{i l}}^{\{t\}}>0 \\
0, & \text { if } \quad \exists i, j \text { s.t. } \dot{S}_{i, t^{\prime}+D_{k j}-D_{i j}}^{\{t\}}=j \\
1, & \text { otherwise. }
\end{array}\right.
$$

Hence

$$
\vartheta_{i}\left(\dot{\mathbf{S}}^{\{t\}}, \dot{\mathbf{x}}\right)=\sum_{k=1}^{N} \sum_{l=1}^{N} \sum_{\delta=0}^{A} C_{k l \delta}\left(\Delta\left(\dot{\mathbf{S}}^{\{t\}}, \dot{\mathbf{x}}\right)\right)
$$

The particular architecture of the $N$-node network being considered deserves to have an appropriate look. Actually, the linear arrangement of the nodes makes the network reach its maximum size, i.e., $A=N-1$. There is no other larger $N$-node network. While nodes are spread over a wide area, two important features should be taken into consideration. On the one hand, this configuration should be taken advantage of to allow simultaneous transmissions as much as possible over both close and faraway branches of the network. On the other hand, the exchanges between distant nodes should be considered. To this end, the distance $D_{k l}$ between a transmitting node $k$ and a destination node $l$ is a key factor to consider in the approximation of the action value function. As opposed to the first category of constraints, this second category is related to the network architecture. Correspondingly, the new expression used to determine the state-action pair value is

$$
\vartheta_{f}\left(\dot{\mathbf{S}}^{\{t\}}, \dot{\mathbf{x}}\right)=D(\dot{\mathbf{x}}) \cdot \vartheta_{i}\left(\dot{\mathbf{S}}^{\{t\}}, \dot{\mathbf{x}}\right)
$$


where $\dot{\mathbf{x}}=(i, j)$ and $D(\dot{\mathbf{x}})=D_{i j}$. Taking into account all constraints inherent to the network under consideration, this measure can be used to approximate the action value function $\dot{Q}$

$$
\dot{Q}\left(\Delta\left(\dot{\mathbf{S}}^{\{t\}}, \dot{\mathbf{x}}\right), \dot{\mathbf{x}}^{\prime}\right)=D(\dot{\mathbf{x}}) \cdot \sum_{k=1}^{N} \sum_{l=1}^{N} \sum_{\delta=0}^{A} C_{k l \delta}\left(\Delta\left(\dot{\mathbf{S}}^{\{t\}}, \dot{\mathbf{x}}\right)\right)
$$

However, the use of distance as a weighting parameter could lead to maintaining the communication between the

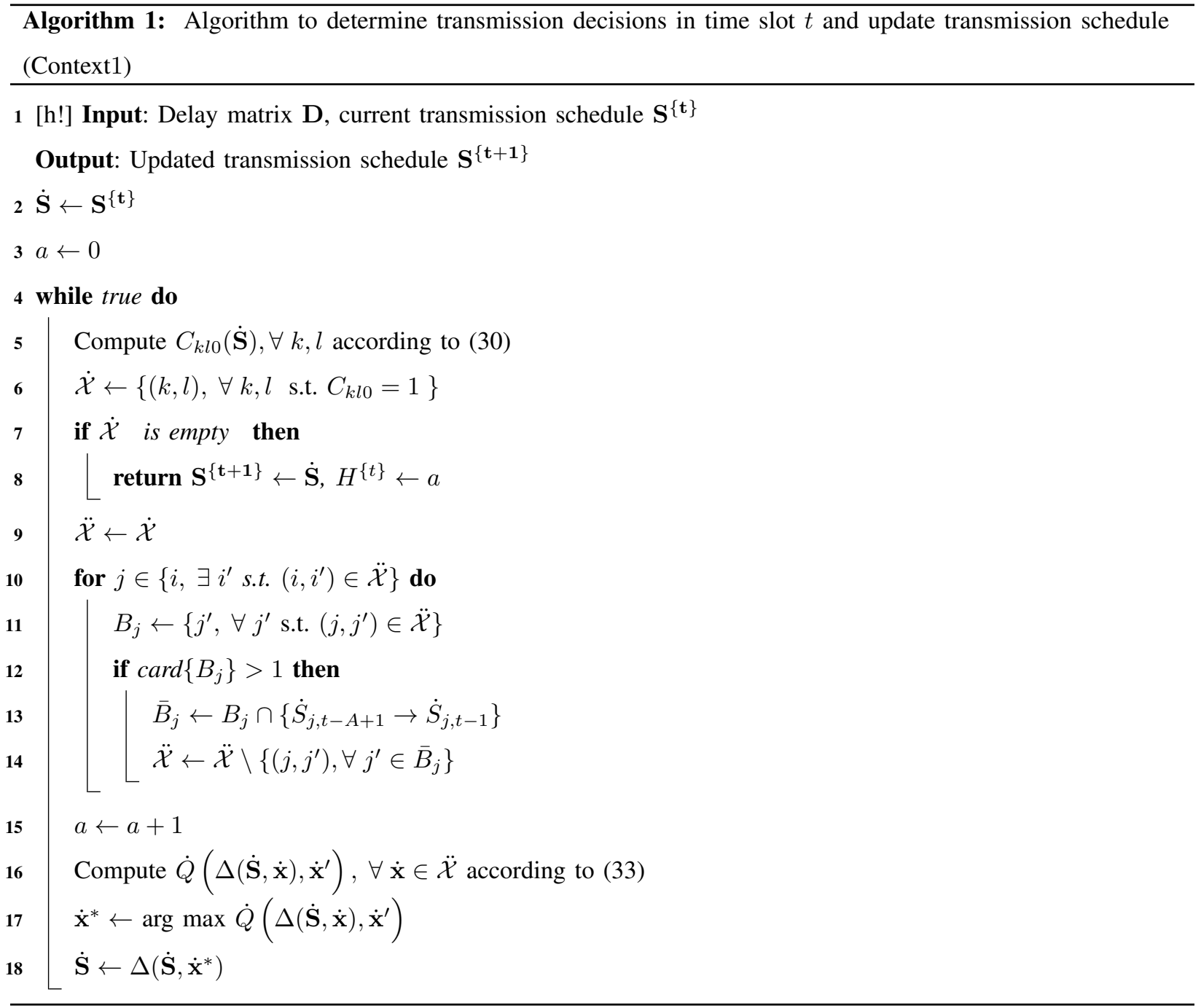

same pairs of remote nodes. Interferences generated thus prevent other nodes from transmitting. We add a weak fairness constraint which helps avoid this issue. Indeed, each transmitting node should diversify its destination nodes over $A$ time slots. In other words, for a certain node $k$, if decision space $\dot{\mathcal{X}}$ contains several opportunities, it is updated before making use of the action value function, i.e., the opportunities to transmit to destination nodes already served by $k$ within the previous $A$ time slots, are excluded. Therefore, the optimal decision $\dot{\mathrm{x}}^{*}$ from the 
updated decision space $\ddot{\mathcal{X}}$ is expressed by

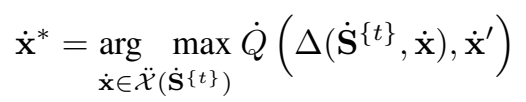

By collecting all the steps discussed above, we end up with an efficient practical algorithm that makes good transmission decisions at time slot $t$ and updates the transmission schedule. A concise description of this algorithm is given as Algorithm 1. Given the partial schedule $\mathbf{S}^{\{\mathbf{t}\}}$, the algorithm goes through the following operations and keeps successively repeating them, until there is no more allowable transmission for that time slot. First, it explores all possible transmission decisions in time slot $t$. Afterwards, the decision space found is updated using the weak fairness constraint of diversifying destination nodes within a time horizon of $A$ time slots. Thereafter, the approximated action value function is used to find the optimal transmission decision. This is a transmission that has little effect on forthcoming transmissions. This algorithm can be seen as an extension of the algorithm proposed in [19], which is well adapted to regular linear networks and even to general linear networks. It requires a short time to result in the throughput-effective schedules presented in section III-A1, for any considered size of the network. These schedules are weakly per-node fair, since every node transmits at least once during one period $T=N$, i.e.,

$$
\sum_{t=0}^{T-1} \mathbb{1}\left(S_{i t}^{(T)}>0\right) \geq 1, \quad \forall i
$$

We summarize results from multiple simulation runs of the algorithm for various $N$ in Fig. 2. The throughput

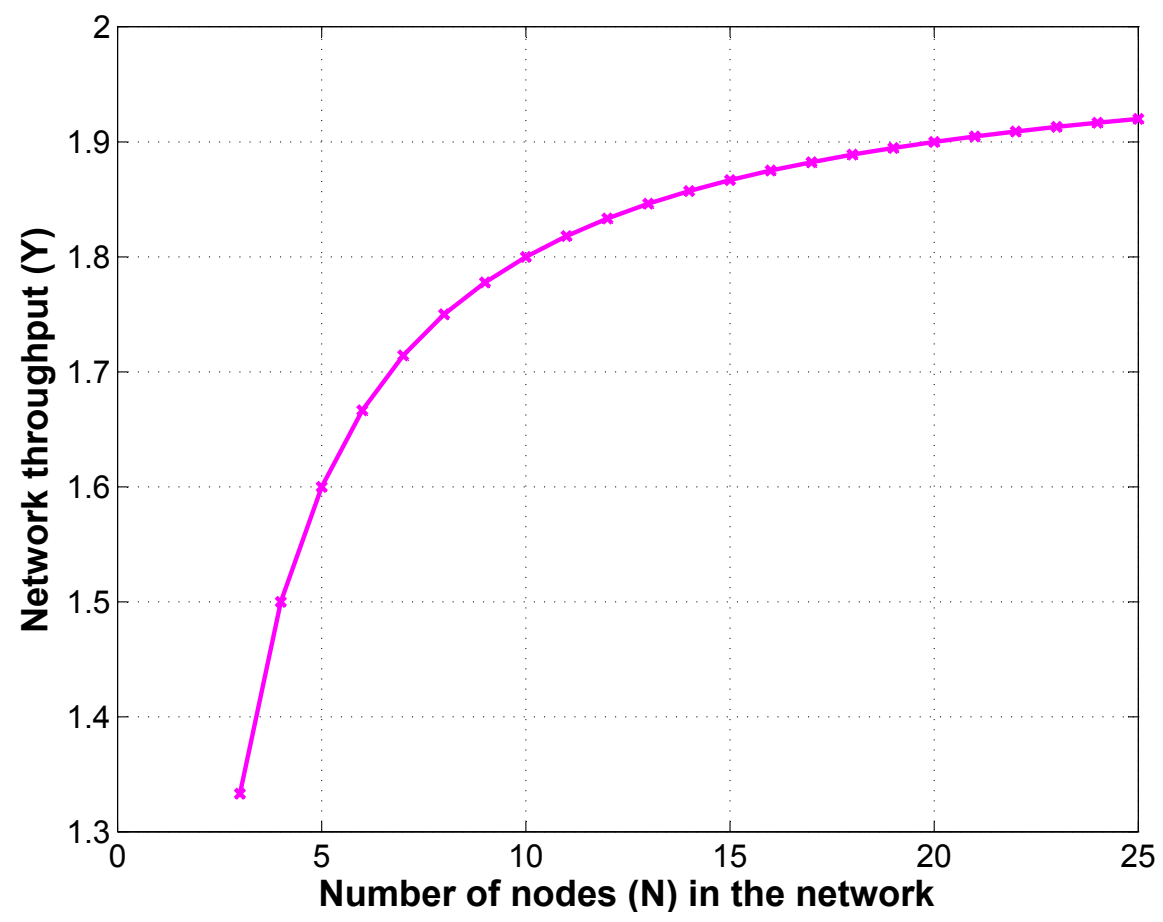

Fig. 2. Network throughput for schedules generated using Algorithm 1 for various $N \leq 25$. The throughput consistently achieves the bound $2-(2 / N)$. 
consistently achieves the bound $2-(2 / N)$. The action value function appropriately approximated combined with the weak fairness constraint make it possible to reach such performance. Algorithm 1 makes it possible to build up a variety of optimal schedules. To this end, one can modify some parameters in the algorithm. For instance, there

are generally more than one entry in the updated decision space $\ddot{\mathcal{X}}$ that correspond to "arg $\max \dot{Q}\left(\Delta(\dot{\mathbf{S}}, \dot{\mathbf{x}}), \dot{\mathbf{x}}^{\prime}\right)$ ". Thus, each one of these entries leads to a new optimal solution and we have just to define the criterion used to choose one of them.

\section{ThroughPUT IN A REGULAR LINEAR NETWORK WITH A SINGLE COLLISION DOMAIN AND BROADCAST}

\section{TRAFFIC}

\section{A. Per-node fair schedule with shortest period}

Lemma 2. In a regular $N$-node linear network with a single collision domain and broadcast traffic, a maximum of two nodes may transmit in the same time slot.

Proof: Consider a regular $N$-node linear network where $i$ is a node transmitting (i.e., broadcasting) at time slot $t$. Thus, all the other nodes in the network will receive from $i$ according to

$$
S_{i t}>0 \quad \Rightarrow \quad S_{l, t+D_{i l}}=-i \quad \forall l, l \neq i
$$

Equivalently, if node $l$ is receiving from node $i$ at time slot $t^{\prime}$, this demonstrates that $i$ has sent a message intended for the whole network

$$
S_{l t^{\prime}}=-i \quad \Rightarrow \quad S_{i, t^{\prime}-D_{i l}}>0
$$

If another node $j$ attempts to transmit in the same time slot $t$, we will face two cases.

Case1: $|i-j|$ is even

Based on (36), the two messages originating from nodes $i$ and $j$ will overlap at node $((i+j) / 2)$ at time slot $t+(|i-j| / 2)$. It follows that the transmission of node $j$ at $t$ leads to interference and consequently is not allowed. To help understand this circumstance, a simple instance where nodes 1 and 5 attempt to transmit at the same time slot is given in the graph in Fig. 3.

Case2: $|i-j|$ is odd

Once more using (36), the two messages originating from nodes $i$ and $j$ will travel in opposite directions between the two neighboring nodes $((i+j-1) / 2)$ and $((i+j+1) / 2)$ at time slot $t+((|i-j|-1) / 2)$. However, they do not overlap at any node in the network. Therefore, the transmission of $j$ at $t$ is allowed. In Fig. 3, a graphical illustration is given of the simple case where nodes 1 and 6 are broadcasting in the 6 -node network at the same time slot.

Let us now consider two nodes $i$ and $j$ transmitting at $t$, where $|i-j|$ is odd. Let us further take into consideration a third node $k, k \neq i$ and $k \neq j$. If $|i-k|$ is even, then $k$ is not allowed to transmit at $t$. If $|i-k|$ is odd, then $|j-k|$ is even since $|i-j|$ is odd. Hence, the transmission of $k$ at $t$ is not permitted. In sum, two is the maximum number of allowable simultaneous transmissions in a regular $N$-node linear network with a single collision domain and broadcast traffic. 


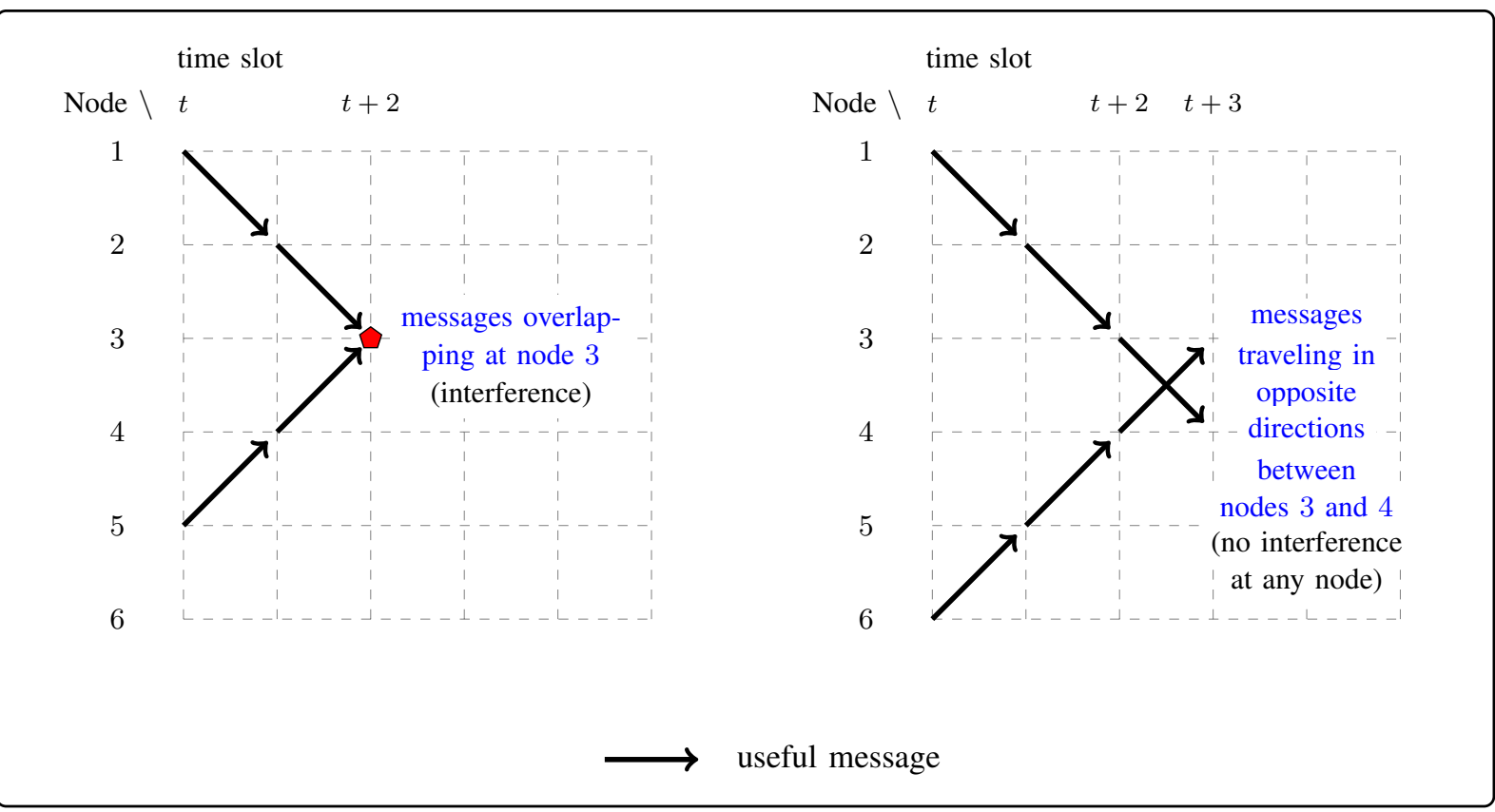

Fig. 3. Depiction of the state of 6 -node network in the case where node-pairs $(1,5)$ and $(1,6)$ are broadcasting at the same time slot

Lemma 3. In a regular $N$-node linear network with a single collision domain and broadcast traffic, if two nodes $i$ and $j$ are transmitting at time slot $t$, there is no transmission allowed at time slots $t+1$ and $t+2$ for any other node $k$ different from $i$ and $j$.

Proof: Consider a regular $N$-node linear network where $i$ and $j$ are transmitting nodes at time slot $t$.

Transmissions at $t+1$

Suppose that there exists a node $k$ that may transmit at $t+1$, where $k \neq i$ and $k \neq j$. Two cases will arise.

Case1: $|i-k|$ is odd, i.e., $\exists l \geq 0$ s.t. $|i-k|=2 l+1$

According to (36), the transmission of node $k$ will give rise to interference in time slot $t+l+1$ at node $i-(l+1)$ if $k<i$, and node $i+(l+1)$ if $k>i$.

Case2: $|i-k|$ is even

Since $|i-k|$ is even and knowing that $|i-j|$ is odd, $|j-k|$ is odd. This is a situation similar to the first case. Therefore, node $k$ will cause interference.

It is concluded that there is no possible transmission at $t+1$.

$\underline{\text { Transmissions at } t+2}$

Suppose now that there exists a node $k$ that may transmit at $t+2$, where $k \neq i$ and $k \neq j$. Three separate cases will arise.

Case1: $k$ has received from both nodes $i$ and $j$

Given the interference constraint, $k$ requires two time slots $(t+1$ and $t+2)$ to receive the two messages sent 
by $i$ and $j$. Besides, given the half-duplex constraint and based on (36), $k$ is not able to transmit at $t+2$ since it is receiving a message.

Case2: $k$ has received from $i$ but has not yet received from $j$

Since $k$ has already received from $i$ before $t+2$, this signifies that $k$ and $i$ are neighboring nodes, i.e., $k=i-1$ or $k=i+1$. Hence, $|j-k|$ is even as long as $|i-j|$ is odd. Consider $l>0$ where $|j-k|=2 l$. As can be deduced from (36), the interference problem will occur in time slot $t+(l+1)$ at node $j+(l+1)$ if $k>j$, and node $j-(l+1)$ if $k<j$. Consequently, the transmission of $k$ at $t+2$ is not possible. The case where $k$ has received from $j$ but not yet from $i$ is exactly the same as the situation that has just been discussed.

Case3: $k$ has not yet received from both nodes $i$ and $j$

If $|i-k|$ is even, the transmission of $k$ is not allowed, as has been shown in the case before. Otherwise, $|i-k|$ is odd. Thus, $|j-k|$ is even and the consequence is still the infeasibility of the transmission of $k$.

It is concluded then that there is no possible transmission at $t+2$.

To sum up, after two simultaneous transmissions at time slot $t$, the next possible transmission will occur at $t+3$.

Theorem 2. In a regular $N$-node linear network with a single collision domain and broadcast traffic, while seeking to grant fair access to all nodes as often as possible (i.e., transmission schedule with the shortest period $T$ ), the maximum achievable throughput is $(N / 2)$ if $N$ is even, and $(N(N-1) /(2 N-1))$ if $N$ is odd (except for $N=3)$.

Proof: Let us consider an $N$-node network where $N$ is even.

Since we are seeking to increase the network throughput, we allow the maximum possible transmissions at the same time slot. From Lemma 2, a maximum of two nodes may transmit at time slot 1 . Let us then grant access to nodes 1 and $N$. Using Lemma 3, the next possible transmissions will occur at $1+3=4$ and so on. Consequently, a transmission scheme that accommodates two transmissions every three time slots will be optimal. Obviously, there will be a need for additional time at the end to allow the last receptions. However, this time should be the most 
advantageous.

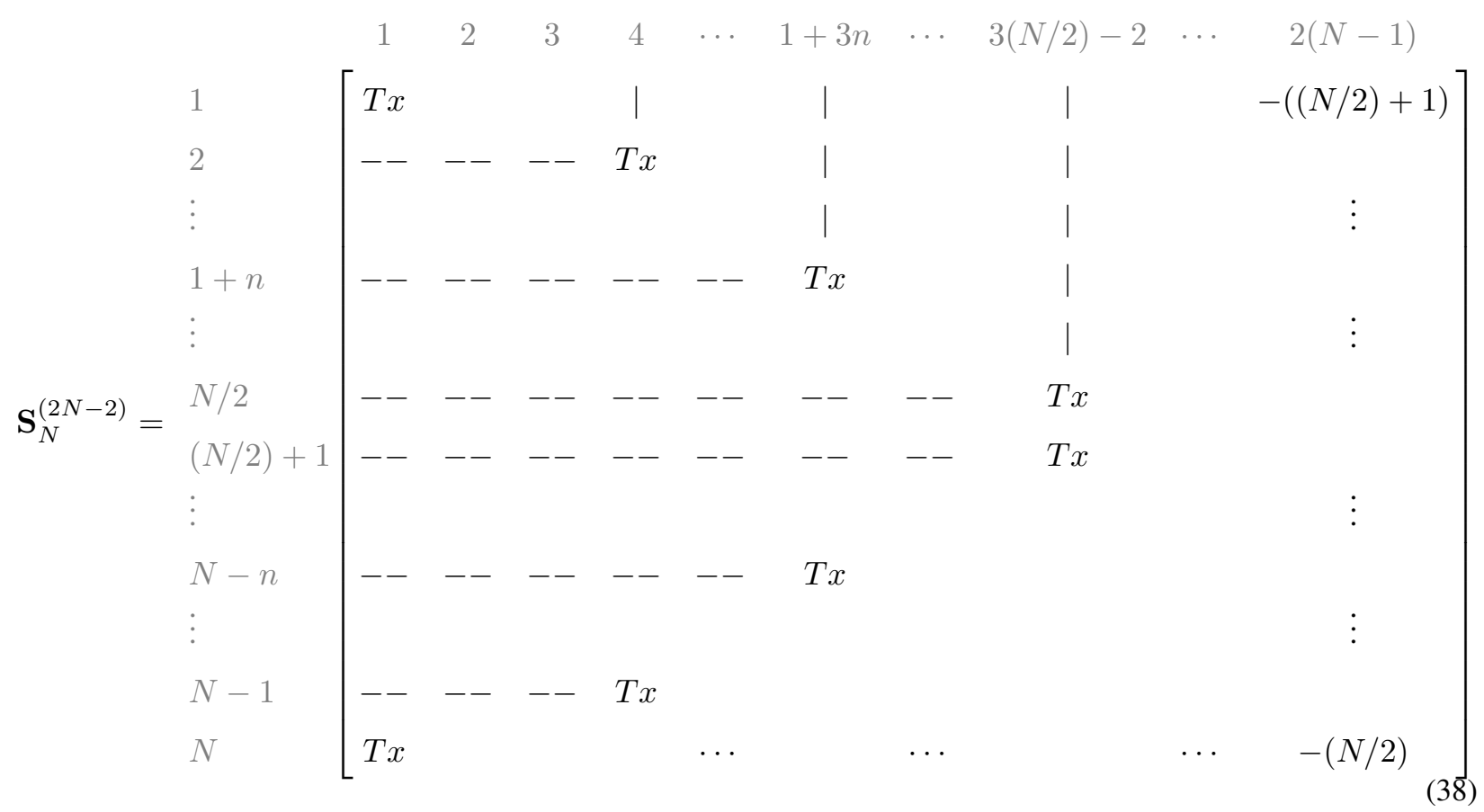

In (38), we introduce the general form of the proposed schedule where we merely highlight the entries corresponding to transmissions denoted by $T x$ and the last receptions. Thereby, after nodes 1 and $N$ at time slot 1 , it is the turn of nodes 2 and $N-1$ to transmit at time slot 4 . Thus, at every time slot $1+3 n(n \geq 0)$, the pair of nodes selected to broadcast is $[1+n, N-n]$, where $n \leq(N / 2)-1$. The transmissions of nodes $(N / 2)$ and $(N / 2)+1$ will occur at $3(N / 2)-2$, and they are the last to transmit. Furthermore, these transmissions require $(N / 2)$ time slots to reach the farthest edge of the network, which is node $N$ when node $(N / 2)$ is transmitting and node 1 when it comes to node $(N / 2)+1$. It is the shortest time that a transmission can take to reach the entire network. With such a scheme where $(N / 2)-1$ pairs of nodes are transmitting every three time slots and the receptions from the last pair take $(N / 2)$ time slots, we end up with a period of $T=3((N / 2)-1)+1+(N / 2)=2(N-1)$. During this period, each node has transmitted once and its message is received by $N-1$ nodes in the network. Subsequently, the throughput achieved is $(N(N-1) / 2(N-1))=(N / 2)$.

In the case where $N$ is odd (except for $N=3$ ), the throughput is $(N(N-1) /(2 N-1))$ and the demonstration is quite similar to that where $N$ is even. Actually, it relies on a transmission scheme allowing two simultaneous transmissions every three time slots, or equivalently two successive transmissions, but still every three time slots. The particularity to note is that since $N$ is odd, except for transmissions in pairs every three time slots, there will be one single transmission for the central node $(N+1) / 2$. It is the last transmission in the schedule and it takes $((N-1) / 2)$ time slots to simultaneously reach both ends of the network, i.e., nodes 1 and $N$. Thus, the corresponding period is $T=3((N-1) / 2)+1+((N-1) / 2)=2 N-1$, in which each node has broadcasted once. Therefore, the throughput achieved is $(N(N-1) /(2 N-1))$.

As regards the particular case where $N=3, \mathbf{S}_{3}^{(6)}$ and $\mathbf{S}_{3}^{\prime(6)}$ exemplify per-node fair schedules and the corre- 
sponding network throughput is 1 . The size of the network is indicated as an index.

$$
\mathbf{S}_{3}^{(6)}=\left[\begin{array}{cccccc}
23 & -2 & 0 & 0 & 0 & -3 \\
13 & -1 & 0 & 0 & -3 & 0 \\
0 & -2 & -1 & 12 & 0 & 0
\end{array}\right] \quad \mathbf{S}_{3}^{\prime(6)}=\left[\begin{array}{cccccc}
23 & 0 & 0 & 0 & -2 & -3 \\
0 & -1 & 0 & 13 & -3 & 0 \\
0 & 0 & -1 & 12 & -2 & 0
\end{array}\right]
$$

To help better understand the schemes designed above, we provide an example for the case where $N$ is even: 6-node network, and another one for $N$ odd: 7-node network. The corresponding per-node fair schedules with the shortest periods are illustrated by $\mathbf{S}_{6}^{(10)}$ and $\mathbf{S}_{7}^{(13)}$, respectively. The entry 23456 in the first row of $\mathbf{S}_{6}^{(10)}$ represents a message originating from node 1 toward destination nodes $2,3,4,5$, and 6 . Note that since each transmission made by a node reaches all the other nodes in the network, a per-node fair schedule is also a per-link fair schedule.

$$
\begin{aligned}
& \mathbf{S}_{6}^{(10)}=\left[\begin{array}{cccccccccc}
23456 & 0 & 0 & 0 & -2 & -6 & 0 & -5 & -3 & -4 \\
0 & -1 & 0 & 13456 & -6 & 0 & -5 & -3 & -4 & 0 \\
0 & 0 & -1 & -6 & -2 & -5 & 12456 & -4 & 0 & 0 \\
0 & 0 & -6 & -1 & -5 & -2 & 12356 & -3 & 0 & 0 \\
0 & -6 & 0 & 12346 & -1 & 0 & -2 & -4 & -3 & 0 \\
12345 & 0 & 0 & 0 & -5 & -1 & 0 & -2 & -4 & -3
\end{array}\right]
\end{aligned}
$$

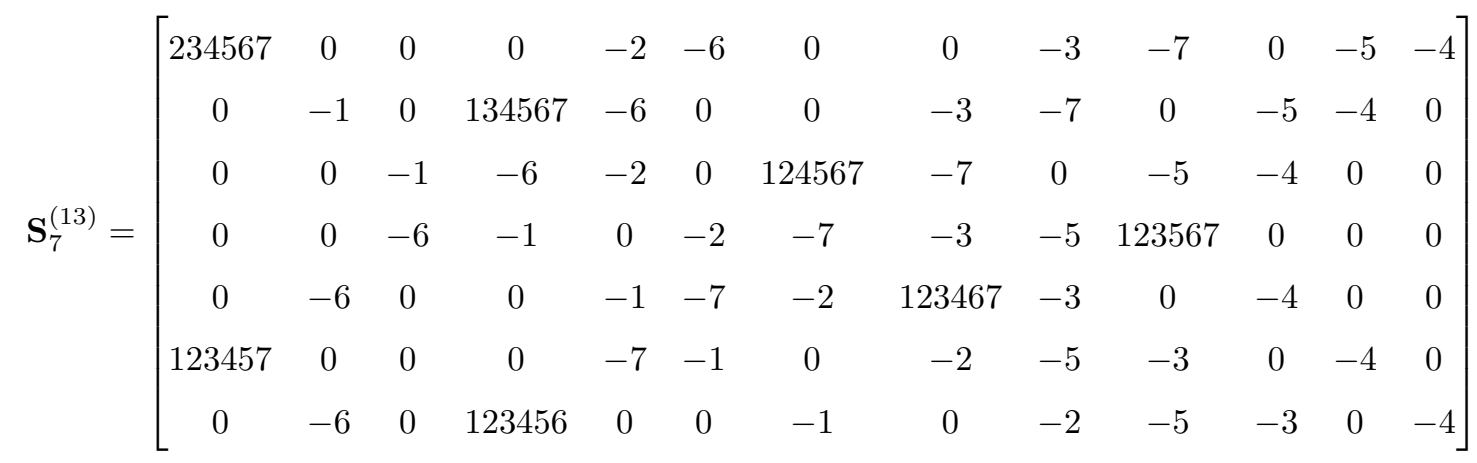

\section{B. Practical algorithm for per-node fair schedule with shortest period}

Unlike the unicast case discussed in the previous section, a node $k$ is allowed to transmit at a certain time slot, if and only if its message can be successfully received by all other nodes. $k$ targets a group of nodes, rather than a single one. Let $L$ denote this destination group composed of all nodes in the network excluding $k$. $\forall l \in L$, if node $l$ is concerned with one of the constraints expressed by the transmission indicator function $C_{k l \delta}$, each node $l^{\prime} \in L \backslash\{l\}$ is also concerned, i.e.,

$$
C_{k l \delta}\left(\dot{\mathbf{S}}^{\{t\}}\right)=0 \Rightarrow C_{k l^{\prime} \delta}\left(\dot{\mathbf{S}}^{\{t\}}\right)=0, \forall l^{\prime} \in L \backslash\{l\}
$$

As regards this first category of constraints, broadcast traffic is more restrictive than unicast delivery. 
By adopting the same problem formulation used in section III-B, the action value function is given by

$$
\begin{aligned}
\dot{Q}(\dot{\mathbf{S}}, \dot{\mathbf{x}}) & =\max _{\dot{\mathbf{x}} \in \dot{\mathcal{X}}(\dot{\mathbf{S}})}\left(H(\dot{\mathbf{x}})+\dot{Q}\left(\Delta(\dot{\mathbf{S}}, \dot{\mathbf{x}}), \dot{\mathbf{x}}^{\prime}\right)\right), \\
& =\max _{\dot{\mathbf{x}} \in \dot{\mathcal{X}}(\dot{\mathbf{S}})} \dot{Q}\left(\Delta(\dot{\mathbf{S}}, \dot{\mathbf{x}}), \dot{\mathbf{x}}^{\prime}\right)+N-1,
\end{aligned}
$$

since $H(\dot{\mathbf{x}})=N-1$ is the reward for the single transmission decided within the considered time slot. Note here that each decision is represented by $\dot{\mathbf{x}}$, that is, one single node (instead of 2-tuple for unicast traffic).

As has been pointed out previously, distance is a key factor in a network with linear architecture. However, since a transmitting node has not one destination node but almost the whole network, the distance from the network central position is considered. An approximation of the action value function $\dot{Q}$ can thus be expressed as

$$
\dot{Q}\left(\Delta\left(\dot{\mathbf{S}}^{\{t\}}, \dot{\mathbf{x}}\right), \dot{\mathbf{x}}^{\prime}\right)=D(\dot{\mathbf{x}}) \cdot \sum_{k=1}^{N} \sum_{l=1}^{N} \sum_{\delta=0}^{A} C_{k l \delta}\left(\Delta\left(\dot{\mathbf{S}}^{\{t\}}, \dot{\mathbf{x}}\right)\right)
$$

where $\dot{\mathbf{x}}=i$ and $D(\dot{\mathbf{x}})=|i-((N+1) / 2)|$, as long as the network central position is identified by $((N+1) / 2)$.

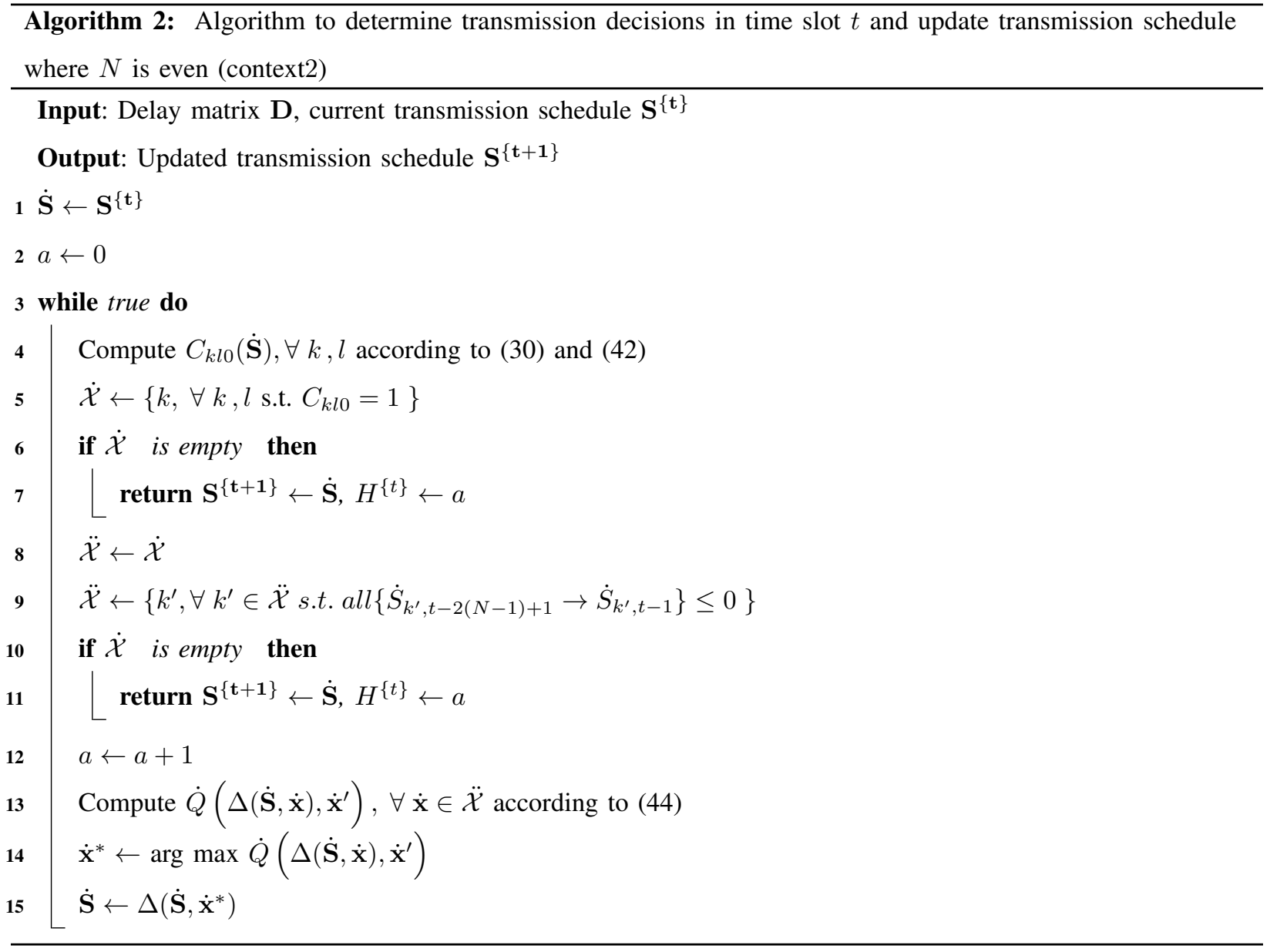

In other respects, since we are interested in per-node fair schedule, each node should, once it has transmitted, allow access to other nodes within a certain time horizon. Likewise, we are interested as well in the shortest period. 
Thus, we add the following constraint to update the decision space $\dot{\mathcal{X}}$ to $\ddot{\mathcal{X}}$ before making use of the action value function: at a time slot $t$, node $k$ is elected to transmit only if it has no transmissions that have occurred during the previous $2(N-1)-1$ time slots in the case where $N$ is even, or during the previous $(2 N-1)-1$ time slots in the case where $N$ is odd. Actually, this time horizon corresponds to one period duration, as shown in the demonstration above.

For $N$ even, a summarization of the algorithm thus designed is presented as Algorithm 2. It makes appropriate transmission decisions at time slot $t$ and updates the transmission schedule. The algorithm in the case where $N$ is odd is almost the same. The only difference is that, at the step corresponding to line 14, for transmission at the same time slot, we favor a pair of distant nodes rather than a pair of neighboring nodes once these pairs are concurrent. The distance is even more essential with the particular architecture of a linear network.

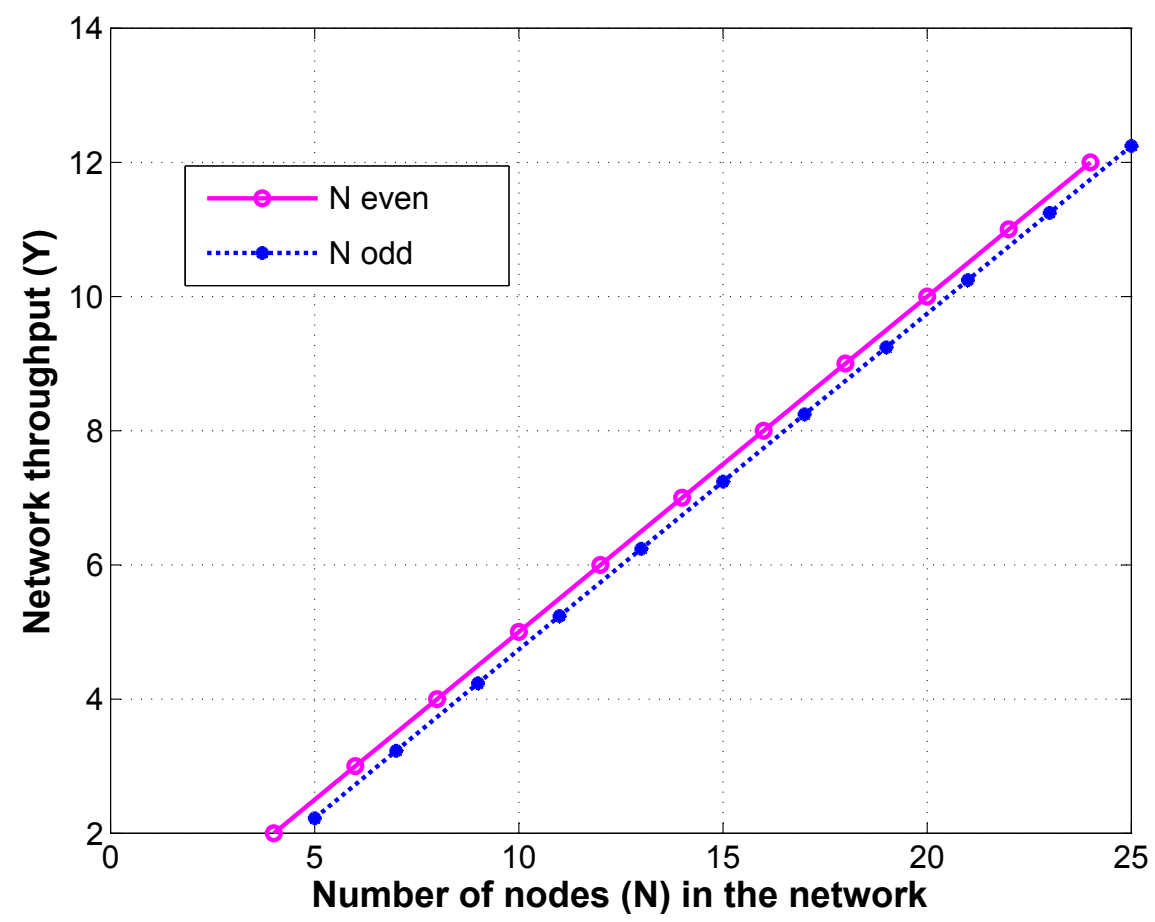

Fig. 4. Network throughput for schedules generated using Algorithm 2 for various $N \leq 25$. The throughput consistently achieves the bounds for both even and odd $N$.

Results from several simulation runs of the algorithm for various $N$ are summarized in Fig. 4. For all the transmission schedules at the output, the throughput consistently achieves the bound $N / 2$ for $N$ even and $N(N-1) /(2 N-1)$ when $N$ is odd.

\section{Multicast transmission schedules with good connectivity and better throughput}

In general, the connectivity in ad hoc networks can be defined as the connection probability of any two nodes via multiple intermediate connections [21]. In our context, since we have one collision domain with reliable physical 
link, only direct information exchange is considered. Keen to exploit the large propagation delays in the UWA environment to achieve better throughput, we provide two illustrations for multicast transmission schedules: 5 -node and 7-node networks. Although the proposed schemes do not offer bidirectional full connectivity as is the case with the broadcast schemes discussed above, the throughput achieved is better with good connectivity. Furthermore, the respective periods are shorter and accordingly more interesting in terms of supporting frequent exchanges in the network. In the notations below, $M$ index is used to designate multicasting while $B$ denotes broadcasting as defined in section IV-A. The size of the network in question is exceptionally specified as index of $\mathbf{S}$.

1) 5-node network: The transmission scheme depicted by $\mathbf{S}_{5}^{(6)}$ ensures good network connectivity during a period of $T_{M}=6$, which is shorter than $T_{B}=9$. Moreover, the corresponding network throughput is $Y_{M}=(7 / 2)$, which is an improvement of more than $57 \%$ compared to $Y_{B}=(20 / 9)$.

$$
\mathbf{S}_{5}^{(6)}=\left[\begin{array}{cccccc}
2345 & -5 & 0 & -4 & -2 & -3 \\
-5 & -1 & -4 & 1345 & -3 & 345 \\
-2 & -4 & -1 & 125 & -2 & -5 \\
1235 & -2 & 0 & -1 & 0 & -2 \\
-2 & -4 & -2 & 123 & -1 & -3
\end{array}\right]
$$

2) 7-node network: The proposed transmission schedule for the 7-node network is depicted by $\mathbf{S}_{7}^{(8)}$. Over the period $T_{M}=8$ (while $T_{B}=13$ ), it performs a network throughput of $Y_{M}=5$. With respect to $Y_{B}=(42 / 13)$, this is an improvement of almost $55 \%$.

$$
\mathbf{S}_{7}^{(8)}=\left[\begin{array}{cccccccc}
234567 & -7 & 0 & -4 & -2 & -6 & 34567 & -5 \\
-7 & -1 & -4 & 134567 & -6 & 0 & -5 & 0 \\
-1 & -4 & -1 & -6 & -2 & -5 & 4567 & -7 \\
1236 & -1 & -6 & -1 & -5 & -2 & -7 & -3 \\
-3 & 0 & -1 & 12347 & -1 & -7 & -2 & 0 \\
12347 & -3 & -4 & -1 & 0 & -1 & 0 & -2 \\
-2 & -6 & -3 & 12345 & -1 & -5 & -1 & 0
\end{array}\right]
$$

\section{Network throughput upper bound}

We have seen that in a regular $N$-node linear network with a single collision domain and broadcast traffic, the transmission schedule achieving the shortest period performs a network throughput of $(N / 2)$ if $N$ is even, and $(N(N-1) /(2 N-1))$ if $N$ is odd. Now, irrespective of the period duration, we are interested in exploring further performance.

Theorem 3. In a regular $N$-node linear network with a single collision domain, broadcast traffic and per-node fair access, the network throughput upper bound is $N-1$.

Proof: Let us consider an $N$-node network where $N$ is even. Since we do not have any restrictions on the period duration, we will make changes to the proposed transmission schedule in section IV-A. At time slot 1 , nodes 
1 and $N$ are transmitting. They will resume transmission every two time slots until they reach $b$ transmissions each. Thus, their $b$ th transmissions occur at time slot $2 b-1$. Afterwards, it is the turn of nodes 2 and $N-1$ to transmit at time slot $2 b+2$ (according to Lemma 3 ). Nodes 2 and $N-1$ will also remain transmitting every two time slots until they reach $b$ transmissions each at time slot $4 b$. To a large extent, every time slot $(2 b+1) n+1$ $(n \geq 0)$, the pair of nodes selected to broadcast $b$ times is $[1+n, N-n]$, while $n \leq(N / 2)-1$. Hence, the transmissions of nodes $(N / 2)$ and $(N / 2)+1$ will occur at $b(N-2)+(N / 2)$, and they are the last to transmit. Their $b$ th transmissions are made at time slot $b N+(N / 2)-2$ and require $(N / 2)$ time slots to reach the entire network. Along these lines, $(N / 2)$ pairs of nodes are transmitting $b$ times. Transmissions from each of $(N / 2)-1$ pairs take $2 b+1$ time slots: $b-1$ times 2 -time-slots in addition to three time slots to allow the transition to the transmissions of the next pair of nodes. However, the receptions from the last pair take $2(b-1)+1+(N / 2)$ time slots. Therefore, the corresponding period is $T=((N / 2)-1)(2 b+1)+2(b-1)+1+(N / 2)=(b+1) N-2$. During this period, each node has transmitted $b$ times and its messages are received by $N-1$ nodes in the network. As a result, the throughput achieved is

$$
Y=\frac{b N(N-1)}{(b+1) N-2}
$$

Let us take an example where $N=4$ and $b=2$. The corresponding transmission schedule is described by $\mathbf{S}_{4}^{(10)}$ and the resulting network throughput is $Y=2.4$, which is greater than $Y=2$ obtained when the period is $T=6$, i.e., $b=1$.

$$
\mathbf{S}_{4}^{(10)}=\left[\begin{array}{cccccccccc}
234 & -4 & 0 & -4 & -2 & -3 & -2 & -3 & 234 & 0 \\
-4 & -1 & -4 & 134 & -3 & 134 & -3 & 0 & 0 & -1 \\
-1 & -4 & -1 & 124 & -2 & 124 & -2 & 0 & 0 & -4 \\
123 & -1 & 0 & -1 & -3 & -2 & -3 & -2 & 123 & 0
\end{array}\right]
$$

By letting $b \longrightarrow \infty$, we end up with an upper bound of $N-1$. Thus,

$$
Y \leq N-1
$$

but we can develop per-node fair schedules with network throughput that may move as close to this upper bound as desired, assuming no constraints on the time extent.

Let us now investigate the possibility of having transmission schedules that achieve the upper bound regardless of the constraints on connectivity and fairness within the network.

If we grant permanent access to one node, the corresponding transmission schedule performs a network throughput of $N-1$. One simple situation such a schedule can refer to, is when a node does not cease to broadcast specific instructions in the whole network. All other nodes are constantly receiving from the broadcasting node. An example 
to highlight this type of schedule is given as $\mathbf{S}_{6}^{(1)}$ for the 6 -node network.

$$
\mathbf{S}_{6}^{(1)}=\left[\begin{array}{c}
23456 \\
-1 \\
-1 \\
-1 \\
-1 \\
-1
\end{array}\right]
$$

If in a different context, the interest is rather to allow two nodes to transmit simultaneously, we would propose transmission schedules where $i$ and $j$ are two transmitting nodes at the same time slot $t$. These transmissions are feasible only if $|i-j|$ is odd (as has been shown in the demonstration of Lemma 2). By way of illustration, we propose for the 6 -node network the schedule $\mathbf{S}_{6<1>}^{(2)}$.

$$
\mathbf{S}_{6<1>}^{(2)}=\left[\begin{array}{cc}
23456 & -6 \\
-6 & -1 \\
-1 & -6 \\
-6 & -1 \\
-1 & -6 \\
12345 & -1
\end{array}\right]
$$

Regarding this second category of schemes where two nodes are transmitting, one may propose a particular design where these transmissions are spread over two successive time slots. For transmitting nodes $i$ and $j$, this is allowed only if $|i-j|$ is even. The transmission schedule $\mathbf{S}_{6<2>}^{(2)}$ is an example for the 6-node network.

$$
\mathbf{S}_{6<2>}^{(2)}=\left[\begin{array}{cc}
23456 & -5 \\
-5 & -1 \\
-1 & -5 \\
-5 & -1 \\
-1 & 12346 \\
-5 & -1
\end{array}\right]
$$

\section{THROUGHPUT IN A REGULAR LINEAR NETWORK WITH PARTIALLY-OVERLAPPING COLLISION DOMAINS}

\section{AND UNICAST TRAFFIC}

Having partially-overlapping collision domains in wireless communication systems is a common phenomenon, when seeking to extend network coverage. This is particularly the case in underwater sensor networks deployed over a large area of interest. A single collision domain may be defined as the geographical area of the network, where transmissions are restricted between all nodes belonging to that collision domain, due to the interference constraint. Actually, the interference range in wireless radio networks is often considered to be approximately twice the transmission range [22], [23]. In the UWA environment, we equivalently assume the same proportions. 


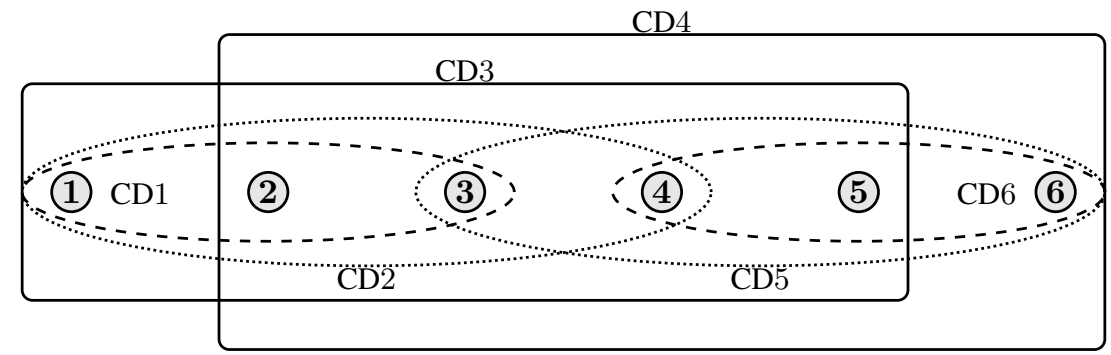

Fig. 5. Partially-overlapping collision domains for $N=6$ with $g=1$

\section{A. Efficient relaying transmission schedule}

When a linear geometry is under consideration, the collision domain is defined as the set of neighboring nodes with a shared medium along which a transmission is propagating, and where resulting interferences terminate. Therefore, a single collision domain is identified with respect to a certain node in the network. We have then $N$ partially-overlapping collision domains. Every collision domain overlaps in part with its adjacent collision domains. Let $g \geq 1$ denote the transmission range of each node, i.e., as regards this constraint, node $i$ can transmit to node $j$ only if

$$
\max (1, i-g) \leq j \leq \min (i+g, N)
$$

In addition, let us use $G_{i}$ to designate the extent of the collision domain within the regular linear $N$-node network, which corresponds to node $i$. Thus,

$$
2 g \leq G_{i} \leq 4 g
$$

Nodes 1 and $N$ at both ends of the network have collision domains of extent $G_{1}=G_{N}=2 g$, while every node $j$ s.t. $2 g<j \leq N-2 g$ has as collision domain extent $G_{j}=4 g$.

We consider in this paper a simple illustration of such a configuration where $g=1$. Regarding packet delivery, unicast traffic is in use. One case of interest may be when each collision domain has to be able to forward the sum of the traffic of its nodes to its immediate neighbor, and so on until reaching the opposite network end. This action can be performed alternatively; in one direction over a specified duration and afterwards in the other direction. The scheme showing different collision domains in the case where $N=6$ and $g=1$ is given in Fig. 5. CD $k$ is used to designate the collision domain relative to node $k$.

Since $g=1$, each node has the opportunity to transmit to its immediate neighbor only, i.e., node $i$ can transmit to node $i+1$ only. Let us first consider the simple case of the 3 -node network. The straightforward way to allow aforementioned transmissions is as follows: at time slot 1 , node 1 transmits to node 2 whereas node 2 transmits to node 3 . Time slot 2 will be reserved for the corresponding receptions. The resulting transmission schedule $\mathbf{S}_{3}^{(2)}$ 
contains 2 transmissions over a period $T=2$. Hence, the network throughput is $Y=1$.

$$
\mathbf{S}_{3}^{(2)}=\left[\begin{array}{cc}
2 & 0 \\
3 & -1 \\
0 & -2
\end{array}\right]
$$

In other respects, there may exist a need to successively transmit two messages for each node instead of one message, as we have seen so far. We thus propose the following transmission schedule depicted by $\mathbf{S}_{3}^{(4)}$ with a period $T=4$.

$$
\mathbf{S}_{3}^{(4)}=\left[\begin{array}{cccc}
0 & 2 & 2 & 0 \\
3 & 3 & -1 & -1 \\
0 & -2 & -2 & 0
\end{array}\right]
$$

The corresponding network throughput is still $Y=1$. More generally, the same throughput can be achieved even in the case where there is a requirement for more successive messages to be transmitted by nodes 1 and 2 . Thus, let $c$ be the number of consecutive messages that every node should transmit. The corresponding schedule $\mathbf{S}_{3}^{(2 c)}$ having a period $T=2 c$, consists of $c$ idle entries in each of the first and third rows, and nonzero entries elsewhere. Let us take the example where $c=5 . \mathbf{S}_{3}^{(10)}$ represents the resulting transmission schedule.

$$
\mathbf{S}_{3}^{(10)}=\left[\begin{array}{cccccccccc}
2 & 2 & 2 & 2 & 2 & 0 & 0 & 0 & 0 & 0 \\
3 & -1 & -1 & -1 & -1 & -1 & 3 & 3 & 3 & 3 \\
-2 & -2 & 0 & 0 & 0 & 0 & 0 & -2 & -2 & -2
\end{array}\right]
$$

Let us now look at larger networks, i.e., $N \geq 4$. Having three adjacent nodes transmitting at the same time slot $t$ is not allowed, as there will be interference on the middle node at $t+1$. Therefore, a transmission schedule with period $T=2$ (like that for the 3 -node network) is no longer interesting. In contrast, the period $T=4$ seems to be more attractive. Indeed, the corresponding transmission schedule does not accommodate more than two adjacent positive entries in the same column. By way of example, $\mathbf{S}_{6}^{(4)}$ describes the schedule for $N=6$ where the throughput is $Y=(5 / 2)$.

$$
\mathbf{S}_{6}^{(4)}=\left[\begin{array}{cccc}
2 & 2 & 0 & 0 \\
3 & -1 & -1 & 3 \\
-2 & -2 & 4 & 4 \\
-3 & 5 & 5 & -3 \\
6 & 6 & -4 & -4 \\
0 & -5 & -5 & 0
\end{array}\right]
$$

When formulating generally, over four time slots, it would be node 1 transmitting two messages successively to node 2 and remaining idle during the other two time slots. Node 2 will in turn transmit two messages consecutively to node 3 , in addition to receiving from node 1 . In this way, node $i$ transmits two messages successively to node $i+1$, and receives from node $i-1$. This operation is performed iteratively until reaching node $N-1$, which is going to transmit twice to node $N$ and receive twice from node $N-2$. Still over the four time slots in question, node $N$ 
receives two messages from node $N-1$ and is idle otherwise. Apart from the first and last rows, which include two idle entries each, the resulting transmission schedule $\mathbf{S}^{(4)}$ consists of nonzero entries. It contains $2(N-1)$ transmissions over a period $T=4$. Hence, the network throughput is $Y=((N-1) / 2)$. Moreover, excluding node $N$, while it is the final destination of the network traffic, $\mathbf{S}^{(4)}$ can be assumed to be per-node fair schedule since

$$
\sum_{t=1}^{4} \mathbb{1}\left(S_{i t}^{(4)}>0\right)=2, \quad \forall i \neq N
$$

In [2], the authors consider transmission protocols with spatial reuse factor $Q$, where each node uses on average, the fraction $1 / Q$ of its time slots for transmission ( $Q$ is an integer for simplicity). In the transmission schedule they proposed and studied, each node has one time slot out of two to use to transmit, to receive or to remain idle. In contrast, if we consider the analogy with our proposed transmission schedule, all time slots are fully used by nodes. In terms of network-oriented metrics, numerical study in [2] shows that it is optimal to operate at $Q=2$. Our proposed transmission schedule satisfies this condition even with fully occupied time slots. Moreover, in [13], for the 11-node network, channel utilization is less than $3 / 2$, while it is equal to 5 for our proposed transmission schedule. Additionally, in the linear topology considered in [14], the authors assume that the transmission range of each node is just one hop and that the interference range is less than two hops. An overall network utilization is a good indicator of network performance. The authors define it as the fraction of time in which the base station is busy receiving messages. With respect to our network modeling, where message duration is set to propagation delay between two neighboring nodes, it has been proven in [14] that, under a per-node fair access constraint with multihop relaying, $1 / 2$ is an asymptotic tight upper bound for the overall network utilization. However, no transmission schedule example achieving this bound is provided. Nevertheless, even if the interference range we adopt in our network modeling is twice the transmission range for each node, our proposed transmission schedule achieves the upper bound in [14], whatever the network size.

\section{B. Effective algorithm for relaying scheme}

The whole $N$-node network consists of several collision domains that are partially-overlapping. Given this context, let us first resume the constraints on transmission under such new conditions. Given the partial schedule $\dot{\mathbf{S}}^{\{t\}}$ at $t$, a single transmission from node $k$ to node $l$ at $t^{\prime} \geq t$ is allowed only if

- node $l$ is within the transmission range of node $k$, i.e., $D_{k l} \leq g$

- it is not a self-transmission, i.e., $l \neq k$

- there is no transmission or reception already planned for node $k$ at $t^{\prime}$, i.e., $\dot{S}_{k, t^{\prime}}^{\{t\}}=0$

- there is no transmission or reception already planned for node $l$ at $t^{\prime}+D_{k l}$, i.e., $\dot{S}_{l, t^{\prime}+D_{k l}}^{\{t\}}=0$

- there is no interference at node $l$ at $t^{\prime}+D_{k l}$, which is originated from any other node $i\left(i \neq k\right.$ and $\left.D_{i l} \leq 2 g\right)$ at $t^{\prime}+D_{k l}-D_{i l}$, i.e., $\nexists i$ s.t. $\left[D_{i l} \leq 2 g\right] \&\left[\dot{S}_{i, t^{\prime}+D_{k l}-D_{i l}}^{\{t\}}>0\right]$

- the transmission from node $k$ will not cause interference on any other node $j\left(j \neq l\right.$ and $\left.D_{k j} \leq 2 g\right)$ at $t^{\prime}+D_{k j}$ while $j$ is receiving a message from node $i\left(D_{i j} \leq g\right)$, i.e., $\nexists i, j$ s.t. $\left[D_{k j} \leq 2 g\right] \&\left[D_{i j} \leq\right.$ $g] \&\left[\dot{S}_{i, t^{\prime}+D_{k j}-D_{i j}}^{\{t\}}=j\right]$ 
This first category of constraints can be summarized using the new transmission indicator function $C_{k l \delta}$ where $\delta=t^{\prime}-t . C_{k l \delta}\left(\dot{\mathbf{S}}^{\{t\}}\right)$ is expressed as

$$
C_{k l \delta}\left(\dot{\mathbf{S}}^{\{t\}}\right)= \begin{cases}0, & \text { if } \quad D_{k l}>g \\ 0, & \text { if } \quad l=k \\ 0, & \text { if } \quad \dot{S}_{k, t^{\prime}}^{\{t\}} \neq 0 \\ 0, & \text { if } \quad \dot{S}_{l, t^{\prime}+D_{k l}}^{\{t\}} \neq 0 \\ 0, & \text { if } \quad \exists i \text { s.t. }\left[D_{i l} \leq 2 g\right] \&\left[\dot{S}_{\left.i, t^{\prime}+D_{k l}-D_{i l}>0\right]}>0\right] \\ 0, & \text { if } \exists i, j \text { s.t. }\left[D_{k j} \leq 2 g\right] \&\left[D_{i j} \leq g\right] \&\left[\dot{S}_{i, t^{\prime}+D_{k j}-D_{i j}}^{\{t\}}=j\right] \\ 1, & \text { otherwise. }\end{cases}
$$

We use the same problem formulation that has been set in section III-B. However, the time horizon to take into consideration becomes $2 g$, since the expiration time of a transmission in the network is equal to $2 g$ time slots. This is the time extent of the current state of the network. In addition to $C_{k l \delta}\left(\dot{\mathbf{S}}^{\{t\}}\right)$, the action value function approximation $\dot{Q}$ relies on the distance parameter, i.e.,

$$
\dot{Q}\left(\Delta\left(\dot{\mathbf{S}}^{\{t\}}, \dot{\mathbf{x}}\right), \dot{\mathbf{x}}^{\prime}\right)=D(\dot{\mathbf{x}}) \cdot \sum_{k=1}^{N} \sum_{l=1}^{N} \sum_{\delta=0}^{2 g} C_{k l \delta}\left(\Delta\left(\dot{\mathbf{S}}^{\{t\}}, \dot{\mathbf{x}}\right)\right)
$$

where $\dot{\mathbf{x}}=(i, j)$ and $D(\dot{\mathbf{x}})=D_{i j}$. Note that throughout the transmission schedule examples introduced above for any communicating nodes pair $(i, j)$, we have $D_{i j}=1$. Hence, the distance factor does not have any impact. Nevertheless, we did identify its utility in the case where $g=2$, while implementing the same action value function approximation.

Furthermore, the action of nodes generating and relaying messages to the network end should be transposed using an additional constraint. Indeed, we update the decision space $\dot{\mathcal{X}}$ to $\ddot{\mathcal{X}}$ before making use of the action value function, in order to orient all transmissions in one direction. In other words, if the flow of messages is planned to be in the direction $\langle$ node $1 \longrightarrow$ node $N\rangle$,

$$
\ddot{\mathcal{X}}=\{(i, j) \in \dot{\mathcal{X}} \quad \text { s.t. } \quad i<j\}
$$

Otherwise, selected 2-tuple $(i, j)$ should be such that $i>j$, when transmissions are oriented in the opposite direction, i.e., $\langle$ node $N \longrightarrow$ node 1$\rangle$.

For $N \geq 4$, the algorithm passing through all the steps outlined above is summarized as Algorithm 3. In addition to delay matrix $\mathbf{D}$ and the current transmission schedule $\mathbf{S}^{\{\mathbf{t}\}}$, the transmission range $g$ is a required input for the algorithm.

Results obtained from numerous simulation runs of the algorithm for various $N$ in Fig. $6 .(N-1) / 2$ is the throughput evermore achieved by all the transmission schedules at the output. 

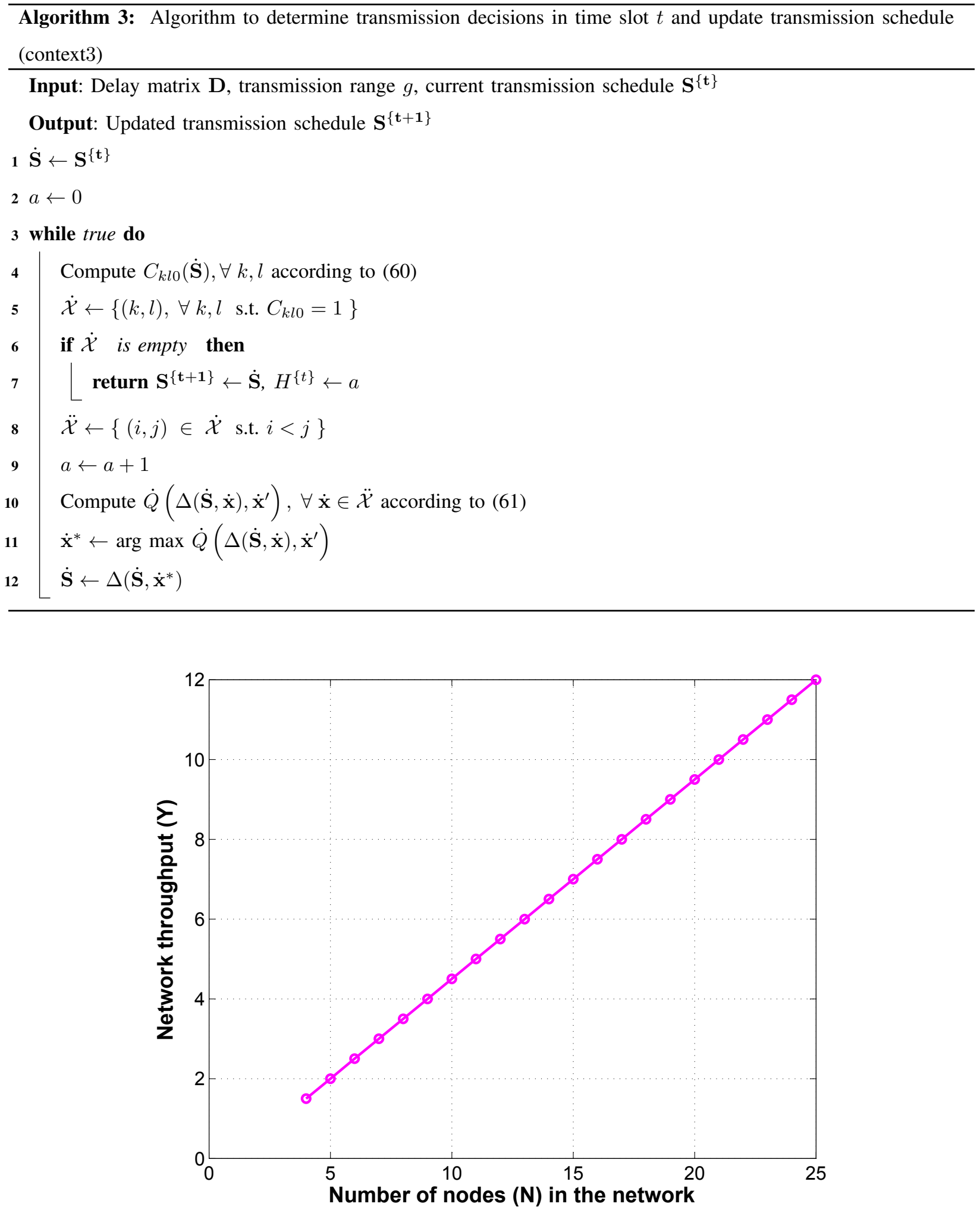

Fig. 6. Network throughput for schedules generated using Algorithm 3 for various $N \leq 25$. The throughput consistently achieves the bound $(N-1) / 2$. 


\section{CONCLUSION}

We considered achievable throughput in the widely used linear network topology. We designed TDMA-based MAC protocols taking advantage of large propagation delays to improve network throughput. We explored three concrete contexts: single collision domain with unicast traffic, single collision domain with broadcast traffic, and partially-overlapping collision domains with unicast traffic. In an $N$-node network, we developed transmission schedules achieving $2-(2 / N)$ as normalized network throughput in the first context. Moreover, we proposed periodic per-node fair schedules with network throughput upper bounds of $N / 2$ and $N-1$ under distinctive fairness constraints in the second context. Likewise, a practical instance of the third context was considered. It depicts a multihop scenario where messages originating at one end of the network are sequentially relayed node-by-node in the direction of the final destination located at the other end of the network. All contexts have been discussed in depth, and with illustrative examples. In addition, computationally efficient algorithms that generate the transmission schedules regardless of the network size, are developed.

The findings of this study help identify the impact of inherent UWA large propagation delays on network throughput in linear topology networks. But above all, the study is a step towards more complex network configuration analysis, such as a grid topology with two or more rows. The combination of network architecture, traffic policy, collision domain extent, and certain fairness constraints should definitely lead to interesting new research problems.

\section{REFERENCES}

[1] S.C. Ergen and P. Varaiya, "PEDAMACS: Power Efficient and Delay Aware Medium Access Protocol for Sensor Networks," IEEE Transactions on Mobile Computing, vol. 5, no. 7, pp. 920-930, July 2006.

[2] Wenyi Zhang, M. Stojanovic, and U. Mitra, "Analysis of a Linear Multihop Underwater Acoustic Network," IEEE Journal of Oceanic Engineering, vol. 35, no. 4, pp. 961-970, Oct. 2010.

[3] B. Peleato and M. Stojanovic, "Distance Aware Collision Avoidance Protocol for Ad-hoc Underwater Acoustic Sensor Networks," IEEE Communications Letters, vol. 11, no. 12, pp. 1025-1027, Dec. 2007.

[4] Xiaoxing Guo, M.R. Frater, and M.J. Ryan, "Design of a Propagation-Delay-Tolerant MAC Protocol for Underwater Acoustic Sensor Networks," IEEE Journal of Oceanic Engineering, vol. 34, no. 2, pp. 170-180, April 2009.

[5] K. Kredo, P. Djukic, and P. Mohapatra, "STUMP: Exploiting Position Diversity in the Staggered TDMA Underwater MAC Protocol," in Proc. IEEE INFOCOM 2009, Rio de Janeiro, Brazil, Apr. 2009.

[6] Hai-Heng Ng, Wee-Seng Soh, and M. Motani, "A Bidirectional-Concurrent MAC Protocol With Packet Bursting for Underwater Acoustic Networks," IEEE Journal of Oceanic Engineering, vol. 38, no. 3, pp. 547-565, July 2013.

[7] Yang Guan, Chien-Chung Shen, and J. Yackoski, "MAC Scheduling for High Throughput Underwater Acoustic Networks," in Proc. IEEE Wireless Communications and Networking Conf. (WCNC), Cancun, Quintana-Roo Mexico, Mar. 2011.

[8] R. Diamant and L. Lampe, "Spatial Reuse Time-Division Multiple Access for Broadcast Ad Hoc Underwater Acoustic Communication Networks," IEEE Journal of Oceanic Engineering, vol. 36, no. 2, pp. 172-185, Apr. 2011.

[9] C. Carbonelli and U. Mitra, "Cooperative Multihop Communication for Underwater Acoustic Networks," in Proc. 1st ACM Int. Workshop Underwater Networks (WUWNet), Los Angeles, CA USA, Sep. 2006.

[10] C.R. Benson, M.J. Ryan, and M.R. Frater, "On the Benefits of High Absorption in Practical Multi-hop Networks," in Proc. OES/IEEE OCEANS Conf., Aberdeen, Scotland, June 2007.

[11] Wanshi Chen and U. Mitra, "Packet Scheduling for Multihopped Underwater Acoustic Communication Networks," in Proc. MTS/IEEE OCEANS Conf., Vancouver, BC Canada, Sept. 2007.

[12] M. Vajapeyam, S. Vedantam, U. Mitra, J.C. Preisig, and M. Stojanovic, "Distributed Space-Time Cooperative Schemes for Underwater Acoustic Communications," IEEE Journal of Oceanic Engineering, vol. 33, no. 4, pp. 489-501, Oct. 2008. 
[13] Yen-Da Chen, Chan-Ying Lien, Sun-Wei Chuang, and Kuei-Ping Shih, "DSSS: A TDMA-based MAC Protocol with Dynamic Slot Scheduling Strategy for Underwater Acoustic Sensor Networks,” in Proc. OES/IEEE OCEANS Conf., Santander, Spain, June 2011.

[14] Yang Xiao, Miao Peng, J. Gibson, G.G. Xie, Ding-Zhu Du, and A.V. Vasilakos, “Tight Performance Bounds of Multihop Fair Access for MAC Protocols in Wireless Sensor Networks and Underwater Sensor Networks," IEEE Transactions on Mobile Computing, vol. 11, no. 10, pp. 1538-1554, Oct. 2012.

[15] V.R. Cadambe and S.A. Jafar, "Degrees of Freedom of Wireless Networks - What a Difference Delay Makes," in Proc. 41st Asilomar Conference on Signals, Systems and Computers (ACSSC), Pacific Grove, CA USA, Nov. 2007.

[16] R. Mathar and G. Bocherer, "On Spatial Patterns of Transmitter-Receiver Pairs That Allow for Interference Alignment by Delay," in Proc. 3rd International Conf. on Signal Processing and Communication Systems (ICSPCS), Omaha, NE USA, Sept. 2009.

[17] R. Mathar and M. Zivkovic, "How to Position n Transmitter-Receiver Pairs in n-1 Dimensions Such That Each Can Use Half of the Channel with Zero Interference from the Others," in IEEE Global Telecommunications Conf. (GLOBECOM), Honolulu, HI USA, Nov. 2009.

[18] F.L. Blasco, F. Rossetto, and G. Bauch, “Time Interference Alignment via Delay Offset for Long Delay Networks," in IEEE Global Telecommunications Conf. (GLOBECOM), Houston, TX USA, Dec. 2011.

[19] M. Chitre, M. Motani, and S. Shahabudeen, “Throughput of Networks With Large Propagation Delays," IEEE Journal of Oceanic Engineering, vol. 37, no. 4, pp. 645-658, Oct. 2012.

[20] Warren B. Powell, Approximate Dynamic Programming: Solving the Curses of Dimensionality, Wiley-Interscience, New York, 2007.

[21] Lei Zhang, Lin Cai, Jianping Pan, and Fei Tong, "A New Approach to the Directed Connectivity in Two-Dimensional Lattice Networks," IEEE Transactions on Mobile Computing, vol. 13, no. 11, pp. 2458-2472, Nov. 2014.

[22] D. Jing, L. Ben, and P.K. Varshney, "Tuning the Carrier Sensing Range of IEEE 802.11 MAC," in Proc. IEEE Global Telecommunications Conf., Dallas, Texas USA, Nov. 2004.

[23] S. Boppana and J.M. Shea, "Overlapped Carrier-Sense Multiple Access (OCSMA) in Wireless Ad Hoc Networks," IEEE Transactions on Mobile Computing, vol. 8, no. 3, pp. 369-383, Mar. 2009. 